\title{
Strong coupling expansion for the conformal Pomeron/Odderon trajectories
}

\author{
Richard C. Brower, ${ }^{a}$ Miguel S. Costa, ${ }^{b}$ Marko Djurić, ${ }^{b}$ Timothy Raben $^{c}$ and Chung-I Tan ${ }^{c}$ \\ ${ }^{a}$ Physics Department, Boston University, \\ Boston MA 02215, U.S.A. \\ ${ }^{b}$ Centro de Física do Porto, Departamento de Física e Astronomia, \\ Faculdade Ciências da Universidade do Porto, Portugal \\ ${ }^{c}$ Physics Department, Brown University, \\ Providence, RI 02912, U.S.A. \\ E-mail: brower@bu.edu, miguelc@fc.up.pt, djuric@fc.up.pt, \\ timothy_raben@brown.edu, chung-i_tan@brown.edu
}

Abstract: From the perspective of AdS/CFT the Pomeron is identified with a Reggeized Graviton, while the Odderons correspond to Reggeized anti-symmetric $A d S_{5}$ Kalb-Ramond tensorfields. In this paper, we consider the strong coupling expansion of the dimension of the leading twist operators dual to these Regge trajectories, $\Delta(j)$, to determine its analytic continuation in $j$ beyond the diffusion limit. In particular, we compute the strong coupling expansion of the intercept to order $\lambda^{-3}$, where $\lambda$ is the t'Hooft coupling, for both the Pomeron, which is $C=+1$ crossing-even, and the "Odderons", which are the leading $C=-1$ crossing-odd Regge singularities. We discuss the spectral curves of the class of single-trace operators to which these string modes couple.

KEYwords: Gauge-gravity correspondence, AdS-CFT Correspondence, Strong Coupling Expansion

ARXIV EPRINT: 1409.2730 


\section{Contents}

1 Introduction 1

2 Conformal Regge representation $\quad 4$

3 Conformal Pomeron $\quad 8$

3.1 Pomeron spin versus anomalous dimensions at strong coupling 9

3.2 Explicit results for $\mathcal{N}=4 \mathrm{SYM} \quad 13$

4 Conformal Odderon $\quad 14$

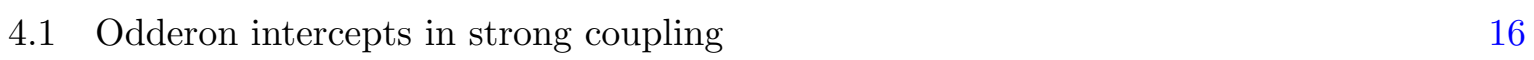

5 Conclusions $\quad 20$

\section{Introduction}

AdS/CFT correspondence [1-4], has provided a useful perspective on several domains of nonperturbative QCD, such as heavy ion collisions, low-energy meson dynamics, and high energy scattering. In particular, at strong coupling the leading Pomeron exchange has been identified as a Reggeized AdS Graviton, in the planar approximation to $\mathcal{N}=4$ Super Yang Mills (SYM) Theory [5].

A crucial observation made in [5] is the role played by the analytic continuation in the $\Delta-j$ plane for anomalous dimensions, $\gamma(j)=\Delta(j)-j-\tau$, for the leading twist operators as a function of $j$ and the 't Hooft coupling $\lambda=g^{2} N_{c}$. In a conformal field theory, the inverse curve in the "Dimension- $j$ " plane, $j(\Delta)$, plays a central role analogous to the traditional Regge pole trajectory $\alpha(t)$ in "Energy- $j$ " plane. As a spectral curve, $\Delta(j)$ has a remarkable symmetry due to conformal invariance: the inverse curve, $j(\Delta)$, is symmetric under $\Delta \leftrightarrow 4-\Delta,{ }^{1}$ with a minimum at $\Delta=2$, as shown in figure 1 for the leading twist-2 spectral curve. At integer $j$ this symmetry relates operators to shadow operators in the conformal field theory. The value of $j$ at this minimum, $j_{0}(\lambda)$, corresponds to the location of the conformal Regge intercept associated with a given spectral curve $\Delta(j)$. In strong coupling, the Pomeron intercept $[5,6]$,

$$
\alpha_{P}=j_{0}(\lambda)=2-\frac{2}{\lambda^{1 / 2}}+O(1 / \lambda)
$$

\footnotetext{
${ }^{1}$ More succinctly stated, conformal symmetry implies a $j$-plane trajectory as a function of $\Delta(\Delta-4)=M_{\text {ads }}^{2} R_{\text {ads }}^{2}$ just as Lorentz invariance implies Regge j-plane trajectories as a function of $\alpha^{\prime} t$. We will occasionally refer to $j(\Delta)$ as the "conformal Reggeon spin", or simply "Reggeon spin".
} 


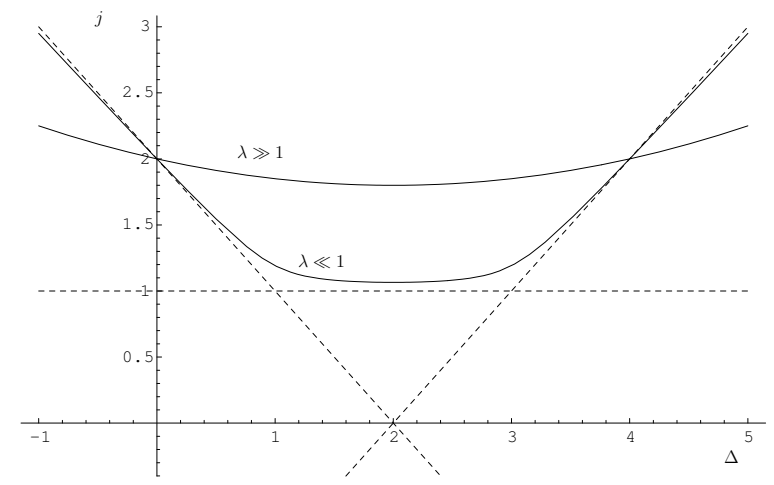

Figure 1. The conformal Regge trajectory reproduced from ref. [5]: schematic form of the $\Delta-j$ relation for twist-2 spectral curve at weak $(\lambda \ll 1)$ and strong coupling $(\lambda \gg 1)$.

was determined by perturbing the spectral curve $\Delta_{P}(j)$ about the graviton at $j=2$ in the super-gravity limit. The same principle can be applied to weak coupling, with the minimum identified with the BFKL Pomeron intercept [7-11]

$$
\alpha_{P}=j_{0}(\lambda)=1+\frac{4 \ln 2}{\pi} \lambda+O\left(\lambda^{2}\right) .
$$

A major challenge in $\mathcal{N}=4 \mathrm{SYM}$ is to determine this intercept $j_{0}(\lambda)$ for all $\lambda$ in the large $N_{c}$ approximation and to apply this analysis to other trajectories [12].

In this paper we apply an expansion procedure for spectral curves, that describes short strings in AdS [13-15], to improve the strong coupling expansion for both the leading conformal Pomeron $(\mathrm{C}=+1)$ and Odderon $(\mathrm{C}=-1)$ trajectories. For the Pomeron this is a simple extension of earlier results [16-19], while the application to the Odderon trajectory [20, 21] is new. For $\mathcal{N}=4 \mathrm{SYM}$, the $\mathrm{C}=+1$ exchange is associated with exchange of local operators with \pm lightcone components,

$$
\mathcal{O}_{P}^{ \pm}(j, k)=\operatorname{Tr}\left[F^{ \pm \perp}\left(D^{ \pm}\right)^{j-2} F_{\perp}^{ \pm} Z^{k}\right]+\cdots,
$$

with $j=2,4,6, \cdots$ and $k=0,1, \cdots$. Here, $Z$ is a scalar field with $\mathrm{SU}(4) \mathrm{R}$-charge. The leading Pomeron has $k=0$, but we extend known results to the case of R-charge exchange with $k \neq 0$. Due to super-symmetry, the analysis can be simplified by relating the relevant spectral curves to that for single-trace operators in the $s l(2)$ sector, symbolically expressed as $\operatorname{Tr}\left[\left(D^{ \pm}\right)^{j-2} Z^{k+2}\right]+\cdots[22,23]$. For the case of the $C=-1$ Odderon trajectory, we shall show how similar techniques can also be applied. ${ }^{2}$

We shall restrict our attention to the pure conformal limit and begin here by briefly describing the Regge limit in the context of conformal field theories [16, 24-26]. Consider the connected component for a four point correlation function of primary operators $\mathcal{O}_{i}$ of dimension $\Delta_{i}$. Defining $x_{i j}=x_{i}-x_{j}$, we have

$$
A\left(x_{i}\right)=\left\langle\mathcal{O}_{1}\left(x_{1}\right) \mathcal{O}_{2}\left(x_{2}\right) \mathcal{O}_{3}\left(x_{3}\right) \mathcal{O}_{4}\left(x_{4}\right)\right\rangle_{c}=\frac{1}{\left(x_{12}^{2}\right)^{\Delta_{1}}\left(x_{34}^{2}\right)^{\Delta_{3}}} F(u, v),
$$

\footnotetext{
${ }^{2}$ Preliminary result was first reported at the "Low-x Workshop", 2013, Rehovot and Eilat, Israel [21].
} 




Figure 2. Conformal compactification of the light-cone showing points taken to null infinity in the Regge limit. In light-cone coordinates $\left(x^{+}, x^{-}, x_{\perp}\right)$ we take $-x_{1}^{+} \sim x_{2}^{+} \rightarrow \infty$ and $-x_{3}^{-} \sim x_{4}^{-} \rightarrow \infty$, keeping $x_{\perp}^{i}$ fixed.

where

$$
u=\frac{x_{12}^{2} x_{34}^{2}}{x_{13}^{2} x_{24}^{2}}, \quad v=\frac{x_{14}^{2} x_{23}^{2}}{x_{13}^{2} x_{24}^{2}}
$$

are the cross ratios and for simplicity we have assumed $\Delta_{1}=\Delta_{2}$ and $\Delta_{3}=\Delta_{4}$. We need to examine the double light-cone limit of vanishing $x_{12}^{2}$ and $x_{34}^{2}$, which corresponds to $u \rightarrow 0$ and $v \rightarrow 1$ in a Minkowski setting. From the perspective of light-cone OPE, this limit can be reached by scaling $x_{1}^{+} \rightarrow \lambda x_{1}^{+}, x_{2}^{+} \rightarrow \lambda x_{2}^{+}, x_{3}^{-} \rightarrow \lambda x_{3}^{-}, x_{4}^{-} \rightarrow \lambda x_{4}^{-}$with $\lambda \rightarrow \infty$, keeping the causal relations $x_{14}^{2}, x_{23}^{2}<0$, as illustrated in figure 2. In a frame where $x_{1 \perp}=x_{2 \perp}$ and $x_{3 \perp}=x_{4 \perp}$, this corresponds to approaching the respective null infinity while keeping the relative impact parameter

$$
b_{\perp}=x_{1 \perp}-x_{3 \perp},
$$

fixed. In terms of the cross ratios, this corresponds to $u \rightarrow 0$ and $v \rightarrow 1$, with $(1-v) / \sqrt{u}$ fixed. Alternatively, defining $u=z \bar{z}$ and $v=(1-z)(1-\bar{z})$ with $z=\sigma e^{\rho}$ and $\bar{z}=\sigma e^{-\rho}$, the precise Regge limit can be specified by $\sigma \rightarrow 0$ for fixed $\rho$.

Using Regge theory the leading behavior of the reduced correlation functions $F$ can be determined by the intercept $j_{0}$, computed from the corresponding leading spectral curve $\Delta(j)$ described earlier, with a general form

$$
F(\sigma, \rho) \approx f(\rho) \frac{\sigma^{1-j_{0}}}{|\ln \sigma|^{3 / 2}}
$$

This is entirely analogous to conventional Regge theory where the spectrum of Regge trajectories determines high energy scattering amplitudes.

The paper is organized as follows. In section 2, we will review the dictionary that translates the Regge description from the language of CFT and OPE into scattering amplitudes in AdS space. This will allow us to proceed directly within the framework of CFT's without explicit recourse to the AdS/CFT correspondence. Nonetheless reference to AdS space can and will be 
made to provide additional intuition. In section 3, we analyze the Pomeron intercept beyond the "diffusion limit", (1.1), leading to a systematic expansion for the Pomeron intercept in $\lambda^{-1 / 2}$. A similar analysis is done for Odderons in section 4. In particular, we clarify how one of the Odderon solutions has intercept that remains fixed at 1 to all orders in $1 / \sqrt{\lambda}$. These results are summarized and discussed in section 5 where we also clarify further the all-coupling formula proposed by Basso in [15], and its possible generalization. We also provide additional comments relating to the weak coupling limit and other issues.

\section{Conformal Regge representation}

While a conformal Regge analysis can be presented entirely in a CFT language [16, 24-26], it is often useful to follow the earlier derivations in invoking a scattering process in AdS [5, 27-30]. Although both approaches are equivalent, they offer separate intuitive frameworks.

Regge theory from CFT partial wave expansion: the t-channel OPE conformal partialwave expansion for the connected component of a 4-point function is given by a sum over conformal blocks,

$$
F(u, v)=\sum_{j} \sum_{\alpha} C_{\alpha, j}^{(12),(34)} G\left(j, \Delta_{\alpha}(j) ; u, v\right) .
$$

For the Regge limit (on the light cone), these are Minkowski conformal blocks, defined with appropriate boundary conditions, or equivalently, as analytic continuation from Euclidean space as explained in refs. [24, 25, 31]. Although, for planar $\mathcal{N}=4$ SYM in the Regge limit, we shall restrict the sum to single-trace conformal primary operators, a completely general representation can be found by introducing basis function for the principle unitary conformal representation and expanding the amplitude as

$$
F(u, v)=\sum_{j} \int_{-\infty}^{\infty} \frac{d \nu}{2 \pi} a(j, \nu) \mathcal{G}(j, \nu ; u, v) .
$$

This group representation combines a discrete sum in the spin $j$ and a Mellin transform in a complex $\Delta$-plane, with $\Delta=2+i \nu$, as explained by Mack [32] and others. ${ }^{3}$ The conformal harmonics, $\mathcal{G}(j, \nu ; u, v)$, are eigen-functions of the quadratic Casimir operator of $\operatorname{SO}(4,2)$. To recover the standard conformal block expansion, it is conventional to close the contour in the lower-half $\nu$-plane, ${ }^{4}$ (equivalently, closing the contour in $\Delta$-plane to the right), picking up only dynamical poles in $a(j, \nu)$, at $\nu(j)=-i(\Delta(j)-2)$. After summing over these pole contributions,

\footnotetext{
${ }^{3}$ We have absorbed factors coming from Plancherel measure, etc., into the partial-wave amplitude $a(j, \nu)$ and will also normalize the conformal harmonics, $\mathcal{G}(j, \nu ; u, v)$, so it eventually leads to conformal blocks with conventional normalization. It has also been demonstrated in [32] how the CFT "Mellin-representation" can be expressed in this group-theoretic form.

${ }^{4}$ Due to conformal invariance, the integrand is even in $\nu$, or, equivalently, symmetric in $\Delta \leftrightarrow 4-\Delta$. The contour can be closed either in the upper or the lower half $\nu$-plane. The poles in the upper half $\nu$-plane corresponds to "shadow" operators.
} 
one arrives at the conformal partial wave expansion (2.1), which also serves as an OPE. These dynamical poles correspond to all allowed conformal primaries, $\mathcal{O}_{\Delta(j)}$, with spin $j$ and dimension $\Delta(j)$.

We are now faced with a discrete sum over spin $j$. A distinguishing feature of the Regge limit is the fact that the conformal harmonics,

$$
\mathcal{G}(j, \nu ; u, v) \sim \sigma^{1-j} \Omega_{i \nu}(\rho), \quad \Omega_{i \nu}(\rho)=\frac{1}{4 \pi^{2}} \frac{\nu \sin (\nu \rho)}{\sinh \rho},
$$

are more and more divergent for increasing $j>1$ as $\sigma \rightarrow 0$. Therefore one cannot take the Regge limit term by term in (2.2). The traditional Regge hypothesis is that this sum can be evaluated by representing the partial wave expansion by the Sommerfeld-Watson transform in the analytic j-plane. For conformal Regge theory, this step leads to a double-Mellin representation [24-26],

$$
F(u, v)=-\int_{-i \infty}^{i \infty} \frac{d j}{2 \pi i} \frac{1 \pm e^{-i \pi j}}{\sin \pi j} \int_{-\infty}^{\infty} \frac{d \nu}{2 \pi} a(j, \nu) \mathcal{G}(j, \nu ; u, v)
$$

where the contour in $j$ is to stay to the right of singularities of $a(j, \nu)$. Note that in $(2.4)$ we must consider separate expressions for even or odd spin values, which will correspond to $C= \pm 1$ contributions respectively. While a direct proof of this $j$-plane representation is lacking for CFT's in general, it has been shown to hold at strong coupling on the basis of the AdS/CFT correspondence for $\mathcal{N}=4 \mathrm{SYM}$ [5] and at weak coupling in the BFKL limit [24, 25]. Moreover it is a natural assumption in order that non-conformal deformation give back the traditional Regge representation. This double-Mellin representation for conformal Regge theory leads to a meromorphic representation in the $\nu^{2}-j$ plane, with poles specified by the collection of allowed spectral curves, $\Delta_{\alpha}(j)$. Still we should emphasize that this conformal "Regge pole hypothesis" is similar but is neither identical or a consequence of the conventional Regge theory. The conventional Regge $j$-plane analyticity with moving singularities in the $j-t$ plane, is replaced by analyticity and moving singularities in this $j-\nu^{2}$ plane. In ref. [5] this distinction is clearly delineated by introducing a confining deformations of the Poincare patch of AdS space which interpolate smoothly between conformal and non-conformal Regge theory.

As an illustration, let us re-consider the $C=+1$ case and focus on the contribution from a single conformal Pomeron pole in $\nu^{2}$,

$$
a(j, \nu)=\frac{r(j)}{\nu^{2}+(\Delta(j)-2)^{2}},
$$

characterized by a spectral curve $\Delta(j)$, with the residue $r(j)$ which vanishes at $j=0,-2,-4, \cdots$. Closing the contour first in the lower-half $\nu$-plane, (2.4) leads to a single-Mellin representation

$$
F(u, v) \approx-\int \frac{d j}{2 \pi i} \frac{1+e^{-i \pi j}}{\sin \pi j} r(j) \sigma^{1-j} \frac{e^{(2-\Delta(j)) \rho}}{\sinh \rho}
$$

where we have taken the limit of $\sqrt{u}=\sigma$ small, with $(1-v) / \sqrt{u} \approx 2 \cosh \rho$ fixed. In this limit, the dominant contribution comes from the right-most singularity in the $j$-plane, which enters 
through $\Delta(j)$. Consider next the spectral curve $\Delta(j)$ for Pomeron exchange and focus on the strong coupling limit where one has $\Delta(j)=2+\sqrt{2} \lambda^{1 / 4} \sqrt{j-j_{0}}$, with $j_{0}$ given by (1.1). Observe that this gives a fixed branch-cut in the $j$-plane. In the limit $\sigma \rightarrow 0$, by pushing the $j$-contour to the left and picking up the contribution from the branch-cut at $j=j_{0},(2.6)$ leads directly to the singular behavior (1.7), as promised. A similar result is also obtained if one considers the weak coupling expansion of the spectral curve $\Delta(j)$, but now $j_{0}$ in (1.7) is given by the BFKL expansion (1.2).

AdS Impact parameter representation: now let us turn to a momentum space treatment for the Regge limit in CFT. Consider the Fourier transform of the connected correlation function defined in (1.4),

$$
(2 \pi)^{4} \delta^{(4)}\left(\sum p_{j}\right) i T\left(p_{1}, p_{2}, p_{3}, p_{4}\right)=\left\langle\mathcal{O}_{1}\left(p_{1}\right) \mathcal{O}_{2}\left(p_{2}\right) \mathcal{O}_{3}\left(p_{3}\right) \mathcal{O}_{4}\left(p_{4}\right)\right\rangle_{c}
$$

The amplitude $T\left(p_{j}\right)$ can be expressed as a function of Mandelstam invariants $s, t$, and $p_{j}^{2}$. The Regge limit corresponds to $s$ large, which defines a light-cone direction, with $t<0$ and $p_{j}^{2}$ fixed. In this limit, the momentum transfer is asymptotically transverse, with $t=\left(p_{1}+p_{2}\right)^{2} \approx-q_{\perp}^{2}$. Using conformal symmetry, it is possible to express the amplitude $T\left(p_{j}\right)$ in an AdS impact parameter representation, which in the Regge limit takes the form [24, 25, 29, 30]

$$
\begin{aligned}
T\left(s, t, p_{i}^{2}\right) & \approx \int \frac{d z}{z^{5}} \frac{d z^{\prime}}{z^{5}} \Phi_{1}\left(z, p_{1}^{2}\right) \Phi_{2}\left(z, p_{2}^{2}\right) \mathcal{G}\left(s, t, z, z^{\prime}\right) \Phi_{3}\left(z^{\prime}, p_{3}^{2}\right) \Phi_{4}\left(z^{\prime}, p_{4}^{2}\right), \\
\mathcal{G}\left(s, t, z, z^{\prime}\right) & =\left(z z^{\prime}\right)^{2} s \int \frac{d^{2} b_{\perp}}{4 \pi^{2}} e^{i q_{\perp} \cdot b_{\perp}} \mathcal{T}(S, L),
\end{aligned}
$$

with $b_{\perp}$ the two-dimensional impact parameter introduced earlier (1.6). The amplitude $\mathcal{T}=$ $\mathcal{T}(S, L)$ encodes all dynamical information and, due to conformal symmetry, depends only on the variables

$$
S=z z^{\prime} s, \quad \cosh L=\frac{z^{2}+z^{\prime 2}+b_{\perp}^{2}}{2 z z^{\prime}} .
$$

The same representation was obtained through direct AdS/CFT considerations [5, 27, 28], via generalized Witten diagrams, string vertex operators, etc., leading to a Regge kernel, $\mathcal{K}\left(s, b_{\perp}, z, z^{\prime}\right)$. Up to irrelevant constants, this kernel is related to the amplitude $\mathcal{T}(S, L)$ by $^{5}$

$$
\mathcal{K}\left(s, b_{\perp}, z, z^{\prime}\right) \sim N^{2}\left(z z^{\prime}\right)^{2} s \mathcal{T}(S, L) .
$$

The Regge limit is now $S \rightarrow \infty$ with fixed $L$. It is important to note that the conformal representation (2.8) of the amplitude is valid for any value of the coupling constant, since it relies only on conformal invariance. However, it is quite natural from the view point of the dual AdS scattering process, where transverse space is precisely a three-dimensional hyperbolic space $H_{3}$, whose boundary is conformal to the physical transverse space $\mathbb{R}^{2}$. The cross ratio

\footnotetext{
${ }^{5}$ The $1 / N^{2}$ dependence in $\mathcal{T}(S, L)$, expected for AdS gravitational interactions, is normally removed from $\mathcal{K}$. In $[27,28]$, a reduced variable $\widetilde{s}=S=z z^{\prime} s$ was used extensively. One occasionally also used $\eta$ or $\xi$, instead of $L$.
} 
$L$ is then identified with the geodesic distance between two points in $H_{3}$ that are separated by $b_{\perp}$ along $\mathbb{R}^{2}$ and have radial coordinates $z$ and $z^{\prime}$. The other cross ratio $S=z z^{\prime} s$ measures the local energy squared of the scattering process in AdS, since $z$ and $z^{\prime}$ define the local AdS scales for each incoming particle. Moreover, the functions $\Phi_{i}\left(z, p_{i}^{2}\right)$ are AdS bulk to boundary propagators with a plane wave source of momentum $p_{i}$ created by the gauge theory operator at the boundary $z \rightarrow 0$. To define on-shell scattering for non-conformal amplitudes, $T(s, t)$, as introduced in ref. [5], one deforms the dual AdS space in the IR, breaking conformal symmetry, and replaces $\Phi_{i}\left(z, p_{i}^{2}\right)$ by normalizable wave functions for "hadronic" (or glueball) eigenstates. ${ }^{6}$

By considering the radial Fourier decomposition in the AdS impact parameter space $\mathrm{H}_{3}$, one can derive a double Mellin representation of the kernel, as done earlier for CFT analysis in a coordinate representation, eq. (2.4). This radial decomposition simply considers harmonic functions $\Omega_{i \nu}$ on $H_{3}$, given by (2.3), which satisfy $\left(\nabla^{2}+1+\nu^{2}\right) \Omega_{i \nu}=0$, where $\nabla^{2}$ is the $H_{3}$ Laplacian. This is equivalent to introducing $\mathbb{R}^{2}$ harmonic functions, $e^{i q_{\perp} b_{\perp}}$, in the standard impact parameter decomposition. The only difference is that now we have a scattering process in AdS space. From a CFT point of view, this representation can also be derived by writing the conformal partial wave decomposition of the amplitude $\mathcal{T}(S, L)$, and then taking the Regge limit. Thus, as before, the $\nu$-integral reflects conformal invariance due to dilatation, and the $j$-integral represents a coherent sum of $t$-channel spin fields, as was done earlier for the OPE sum via a Sommerfeld-Watson transform. To be more explicit, since the Pomeron/Odderon kernels receive contributions respectively from all even/odd spins, these kernels can be expressed as

$$
\mathcal{K}_{ \pm}\left(s, b_{\perp}^{2}, z, z^{\prime}\right)=-\left(z z^{\prime}\right) \int \frac{d j}{2 \pi i} \frac{1 \pm e^{-i \pi j}}{\sin \pi j} \int_{-\infty}^{\infty} \frac{d \nu}{2 \pi} S^{j} G_{ \pm}(j, \nu) \Omega_{i \nu}(L) .
$$

This representation is a consequence of conformal invariance, which must next be supplemented by dynamics, i.e., specifying the Pomeron/Odderon propagator $G(j, \nu)$.

By examining Witten diagrams for exchanging spin- $j$ fields in the Regge limit and also their string duals, one is led to $G(j, \nu)$ having a simple pole in the $\nu^{2}$-plane, determined by the spectral curve $\Delta(j)$ associated with these fields, exactly as in coordinate treatment $(2.5)$. Here $G(j, \nu)$ can be related to the $\nu$-transform of a transverse scalar bulk-bulk propagator with an effective $j$-dependent AdS mass. The residue at this pole can be related to the local AdS coupling of the exchanged fields to the external states. Upon closing the $\nu$-contour, one picks up a factor $G\left(j, b_{\perp}, z, z^{\prime}\right) \sim e^{-(\Delta(j)-2) L} / \sinh L$. Finally, for Pomeron(Odderon) exchange, by identifying the $j$-plane branch-point at $j_{0}$ associated with the Pomeron(Odderon) spectral curve, from (2.11) one has for $S$ large, by pulling the contour to the left,

$$
\mathcal{K}\left(s, b_{\perp}^{2}, z, z^{\prime}\right) \approx\left(z z^{\prime}\right) \tilde{f}(L) \frac{S^{j_{0}}}{|\ln S|^{3 / 2}},
$$

just as in the coordinate representation (1.7).

\footnotetext{
${ }^{6}$ Note that at weak coupling the product of wave functions $\Phi_{1} \Phi_{2}$ and $\Phi_{3} \Phi_{4}$ are similarly replaced by the dipole parton distributions for the external particles [25], so that this impact parameter representation (2.8) is maintained.
} 
Regge dictionary for CFT: we have therefore two representations of the correlation function in the Regge limit. One derived from the CFT analysis in position space $F(u, v)$, given by (2.4), and another from a computation in momentum space with a clear geometrical interpretation as a scattering process in AdS, given by (2.11). This establishes a dictionary, where, in the Regge limit,

$$
\begin{aligned}
F(u, v) & \leftrightarrow \mathcal{T}(S, L)=N^{-2}\left(z z^{\prime}\right)^{-2} s^{-1} \mathcal{K}\left(s, b_{\perp}^{2}, z, z^{\prime}\right), \\
\sigma=\sqrt{u} & \leftrightarrow S^{-1}=\left(z z^{\prime} s\right)^{-1}, \\
\cosh \rho \approx \frac{1-v}{2 \sqrt{u}} & \leftrightarrow \cosh L=\frac{b_{\perp}^{2}+z^{2}+z^{\prime 2}}{2 z z^{\prime}} .
\end{aligned}
$$

Although it is possible to carry out a more formal analysis in establishing this equivalence, we will not pursue this here [26]. It suffices to emphasize the exact equivalence of the two approaches to identify the spectral curve, $\Delta(j)$ in figure 1 , which serves as the common link between them.

\section{Conformal Pomeron}

The Pomeron spectral curve $\Delta_{P}(j)$ in the strong coupling limit, figure 1 , can be obtained by an intuitive derivation based on a flat-space leading closed-string linear trajectory. Through AdS/CFT, this simple result can be understood as a perturbation about the traceless-transverse graviton mode, $\nabla^{2} h_{M N}=0$, with $j=2$ and $\Delta=4$ in the $\lambda=\infty$ super-gravity limit. Here $\nabla^{2}$ is the tensor Laplacian on $A d S_{5}$. Let us now consider the limit of $j \rightarrow 2$ and $\lambda \rightarrow \infty$ with $\sqrt{\lambda}(j-2)$ fixed. This limit can be understood by introducing a Reggeon vertex operator, $\mathcal{V}^{ \pm}$, on the string world sheet in a weakly curved target $A d S_{5} \times S^{5}$ space [5]. This Reggeon vertex operator depends on $(j, \nu, t)$ for the $O(4,2)$ Casimir, and on $k=\tau-2$ in case we wish to consider exchange of $\mathrm{SO}(6) R$-charge. The effect of the Reggeon operator is to resum the exchange of all modes in the leading Regge trajectory with even positive integral spins, which leads to the effective Regge spin

$$
j=2-\frac{\tau^{2}+\nu^{2}}{2 \sqrt{\lambda}} .
$$

Then the world-sheet Virasoro on-shell condition, $L_{0} \mathcal{V}^{ \pm}=\bar{L}_{0} \mathcal{V}^{ \pm}=\mathcal{V}^{ \pm}$, establishes the relation

$$
\Delta_{P}(j, \tau)=2+i \nu
$$

where $\Delta_{P}(j, \tau)$ is the continuation of the anomalous dimension curve for the exchanged gauge theory operators in the leading Regge trajectory $\mathcal{O}_{P}^{ \pm}(j, \tau)$ given in (1.3). By following this procedure, one relates the exchange of AdS higher spin fields to the dual gauge theory operators with spectral curve $\Delta_{P}(j, \tau)$.

In the above double limit the Regge spin has a quadratic dependence in the dimension $\nu$, also known as the diffusion limit (in a weak coupling expansion we may also consider such a diffusion limit). The terminology stems from the fact that the kernel in momentum space takes 
on a diffusion form at $t=0$, with diffusion time $\ln \left(z z^{\prime} s\right)$ fixed by the AdS energy $S=z z^{\prime} s$ [5]. In this limit we can already observe that the spectral curve $\Delta_{P}(j, \tau)$ has a branch point at $j_{0}$,

$$
\Delta_{P}(j)=2+\sqrt{2 \lambda^{1 / 2}} \sqrt{j-j_{0}} .
$$

where

$$
j_{0}=2-\frac{\tau^{2}}{2 \sqrt{\lambda}}
$$

which generalizes (1.1) for the case of twist $\tau$.

Beyond the diffusion limit (3.1) the Reggeon spin $j=j(\nu, \tau)$ admits the strong coupling expansion

$$
j(\nu, \tau)=2-\frac{\tau^{2}+\nu^{2}}{2 \sqrt{\lambda}}\left(1+\sum_{n=2}^{\infty} \frac{\tilde{j}_{n}\left(\nu^{2}, \tau\right)}{\lambda^{(n-1) / 2}}\right),
$$

which is a simple generalisation to arbitrary twist $\tau$ of the results presented in $[16,24]$ (such that at infinite coupling, for $j=2$, the dimension of the operator is given by the protected value of $2+\tau)$. Notice that $j(\nu, \tau)$ must be an even function of $\nu$ to implement the symmetry property $\Delta_{P}(j, \tau) \leftrightarrow 4-\Delta_{P}(j, \tau)$. The function $\tilde{j}_{n}\left(\nu^{2}, \tau\right)$, defined for $n \geq 2$, is a polynomial of degree $n-2$,

$$
\tilde{j}_{n}\left(\nu^{2}, \tau\right)=\sum_{k=0}^{n-2} c_{n, k} \nu^{2 k}
$$

with $\tau$-dependent coefficients $c_{n, k}$. This follows from the requirement that the AdS amplitude has a well defined flat space limit [24]. Consistency with the strong coupling expansion of the spectral curve $\Delta_{P}(j, \tau)$ further restricts this polynomials to have smaller degree [16], more precisely, for $n \geq 4$

$$
c_{n, k}=0 \text { for }\left[\frac{n}{2}\right] \leq k \leq n-2,
$$

as also confirmed in [18].

Eq. (3.5) corresponds to an expansion for the Reggeon spin about the symmetry point $\Delta=2,\left(\nu^{2}=0\right)$, in the strong coupling limit, subject to the constraint that $j=2$ at $\nu^{2}=-\tau^{2}$. In the next subsection we review recent results for the strong coupling expansion of the spectral curve $\Delta_{P}(j, \tau)$ about $j=2$ that will allow us to compute the pomeron spin $j(\nu)$, i.e., the inverse of the spectral curve $\Delta(j)$, with $\Delta$ and $\nu$ related by (3.2), beyond the diffusion limit at arbitrary twist $\tau$. The discussion leads to a unified picture that can also be applied to the Odderon Regge trajectories, in section 4 .

\subsection{Pomeron spin versus anomalous dimensions at strong coupling}

Much attention has been paid in recent years to the study of anomalous dimensions for composite operators of $\mathcal{N}=4 \mathrm{SYM}$. Because of supersymmetry, many related operators share the same anomalous dimensions. It is generally believed that, due to integrability [33], scaling dimensions for gauge invariant operators can be efficiently calculated for all 't Hooft coupling, in the large$N$ planar limit, via the so-called TBA/Y-system and its generalizations [34]. These operators 
and their cousins can be treated as generalized Heisenberg spin chains. For instance, the weakcoupling one-loop anomalous dimension $\gamma(S, \tau)$ of single-trace operators in the $\operatorname{sl}(2)$ sector, symbolically expressed as $\operatorname{Tr}\left[D_{ \pm}^{S} Z^{\tau}\right]+\cdots$, can be calculated explicitly, with $\Delta(S, \tau)=S+\tau+$ $\gamma(S, \tau)$ their scaling dimension. However, beyond one-loop, and particularly for short operators ( $S$ and $\tau$ small), analytic solutions have been difficult to obtain. For strong coupling, conformal dimensions can be calculated semi-classically in a world-sheet sigma model approach around soliton solutions [35-37], as semi-classical treatment for GKP strings. This leads to a strong coupling loop-expansion, with $1 / \sqrt{\lambda}$ playing the role of $\hbar$,

$$
\Delta=\lambda^{1 / 4}\left(\delta_{0}+\frac{\delta_{1}}{\lambda^{1 / 2}}+\frac{\delta_{2}}{\lambda}+\frac{\delta_{3}}{\lambda^{3 / 2}}+\frac{\delta_{4}}{\lambda^{2}}+\cdots\right)
$$

with $S$ and $\tau$ dependence entering the $\ell$-loop contribution $\delta_{\ell}$ through a scaling hypothesis. ${ }^{7}$ However, calculation beyond 1-loop is impractical. In most approaches of this type, the emphasis has been on long strings.

One analysis of particular interest to us is the expansion for the spectral curve about the point $S=0$,

$$
\Delta_{Z}(S, \tau)=\tau+\alpha_{1}(\tau, \lambda) S+\alpha_{2}(\tau, \lambda) S^{2}+\alpha_{3}(\tau, \lambda) S^{3}+\cdots
$$

where $\Delta_{Z}(0, \tau)=\tau$, since the operator is $1 / 2$-BPS and its dimension is protected. The form of the "slope function", $\alpha_{1}(\lambda, \tau)$, has recently been conjectured by Basso for all 't Hooft coupling [15] (see also [40,41]), which can be expressed in a compact form in terms of Bessel functions,

$$
\alpha_{1}(\lambda, \tau)=\frac{\sqrt{\lambda}}{\tau} Y_{\tau}(\sqrt{\lambda})
$$

for all $\lambda$, with $Y_{\tau}(x)=I_{\tau}^{\prime}(x) / I_{\tau}(x)$, where $I_{\tau}(x)$ is the $\tau$-th modified bessel function. At weak coupling, $\alpha_{1}(\lambda, \tau)=1+O(\lambda)$, and at the strong coupling, $\alpha_{1}(\lambda, \tau)=\sqrt{\lambda} / \tau+O(1 / \sqrt{\lambda})$. This result was first derived for $\tau=2$ as a solution to the "asymptotic Bethe ansatz" (ABA) equations. It has been argued in [15], with further support in [40, 41], that this holds for all $\tau>2$, for the configurations with "minimum mode numbers". ${ }^{8}$ More recently, the second coefficient, $\alpha_{2}(\lambda, \tau)$, has also been calculated numerically, but it is not possible at this moment to express it in a closed form in terms of elementary functions [19, 42]. Due to super-symmetry, it is known that the Pomeron spectral curve ${ }^{9}$ is directly related to $\Delta_{Z}(S, \tau)$ at $\tau=2$ by $[22,23]$

$$
\Delta_{P}(j)=2+\Delta_{Z}(j-2,2) .
$$

Therefore, these recent analyses, appropriately generalized, can be applied to our study of conformal Pomeron and Odderon, particularly in the large $\lambda$ limit.

\footnotetext{
${ }^{7} \delta_{\ell}$ is assumed to be a function of $\mathcal{S}=S / \sqrt{\lambda}$ and $\mathcal{T}=\tau / \sqrt{\lambda}$. Occasional discussions for small $S$ and/or small $\tau$ are typically based on extrapolation under this scaling hypothesis. As such, less attention has been paid in the past to the symmetry property in $\Delta$. For an alternative but related study, see [38, 39].

${ }^{8}$ We will return to a discussion on this and related issues in sections 4 and 5 .

${ }^{9}$ In order to avoid notational confusion, in what follows, instead of $S$, we shall switch to $j=S+2$, e.g., for the $\operatorname{sl}(2)$ sector, we have $\operatorname{Tr}\left[D_{ \pm}^{j-2} Z^{\tau}\right]$, instead of $\operatorname{Tr}\left[D_{ \pm}^{S} Z^{\tau}\right]$.
} 
For planar $\mathcal{N}=4 \mathrm{SYM}$, it is possible to generalize our discussion for the Pomeron spectral curve to include CFT operators with $R$ charges, which through AdS/CFT, amounts to allow fluctuations in $S^{5}$. For our purpose, as already described in (1.3), the relevant CFT operators are

$$
\mathcal{O}_{P}(j, \tau)=\operatorname{Tr}\left[F_{\mu \sigma} D_{\rho_{1}} \cdots D_{\rho_{s}} F_{\sigma \nu} Z^{\tau-2}\right]+\cdots,
$$

with $\tau \equiv 2+k \geq 2$. For the leading Regge singularity, we will be dealing with the lightcone components $\operatorname{Tr}\left[F_{ \pm \perp} D_{ \pm} \cdots D_{ \pm} F_{\perp \pm} Z^{\tau-2}\right]+\cdots$. The generalized Pomeron spectral curve $\Delta_{P}(j, \tau)$ can be expanded using (3.11) and (3.9) around $j=2$, leading to

$$
\Delta_{P}(j, \tau)=2+\tau+\alpha_{1}(\lambda, \tau)(j-2)+\alpha_{2}(\lambda, \tau)(j-2)^{2}+\alpha_{3}(\lambda, \tau)(j-2)^{3}+\cdots .
$$

where (3.10) applies.

Because of the symmetry under $\Delta_{P}(j, \tau) \leftrightarrow 4-\Delta_{P}(j, \tau)$, we again require the function $\Delta_{P}(j, \tau)$ to have a square-root singularity at $j_{0}(\tau)$, with $j_{0}(\tau)=2-O\left(\lambda^{-1 / 2}\right)$. This branch point renders the expansion (3.13) with a radius of convergence which vanishes as $\lambda^{-1 / 2}$, leading to expansion coefficients which grow as $\alpha_{n} \sim O\left(\lambda^{n / 2}\right)$. Nevertheless, a convergent expansion can be achieved by considering the symmetric combination $\left(\Delta_{P}(j, \tau)-2\right)^{2}=-\nu^{2}$, for which this square-root branch point is absent. This in turn leads to a convergent expansion in the strong coupling limit,

$$
\left(\Delta_{P}(j, \tau)-2\right)^{2}=\tau^{2}+\beta_{1}(\lambda, \tau)(j-2)+\beta_{2}(\lambda, \tau)(j-2)^{2}+\beta_{3}(\lambda, \tau)(j-2)^{3}+\cdots,
$$

where $\beta_{1}(\lambda, \tau)=2 \tau \alpha_{1}(\lambda, \tau), \beta_{2}(\lambda, \tau)=\alpha_{1}(\lambda, \tau)^{2}+2 \tau \alpha_{2}(\lambda, \tau)$, etc. Based partly on semiclassical analysis of GKP strings [35-37], one expects a radius of convergence of the order $O\left(\lambda^{1 / 2}\right)$. Consistency with (3.3) and the existence of a smooth super-gravity limit then require that $\beta_{n} \sim O\left(\lambda^{(2-n) / 2}\right)$, so that each coefficient $\beta_{n}(\lambda)$ in turn admits an expansion,

$$
\beta_{n}=2 \lambda^{\frac{2-n}{2}}\left(b_{n, 0}+\frac{b_{n, 1}}{\lambda^{1 / 2}}+\frac{b_{n, 2}}{\lambda}+\frac{b_{n, 3}}{\lambda^{3 / 2}}+\cdots\right),
$$

where we have taken the factor of 2 out so that later on the expansion will be normalized with $b_{1,0}=1$, and the coefficients $b_{n, m}$ are in general $\tau$-dependent. The viability of the strong coupling treatment done by Basso in [15] relies on this rapidly convergent expansion. ${ }^{10}$ It is now a simple exercise to check that, since $\beta_{n}$ starts at order $\lambda^{(2-n) / 2}$, we have

$$
\left(\Delta_{P}(j, \tau)-2\right)^{2}=\tau^{2}+2 \sqrt{\lambda}(j-2)\left(1+\sum_{k=1}^{\infty} \lambda^{-\frac{k}{2}} H_{k}(j-2, \tau)\right),
$$

with

$$
H_{k}(j-2, \tau)=\sum_{n=0}^{k} b_{n+1, k-n}(j-2)^{n} .
$$

\footnotetext{
${ }^{10}$ It is worth noting that the expansion for $\beta_{1}$ in $1 / \sqrt{\lambda}$ can be identified with the GKP-loop expansion, i.e., the coefficient $b_{1, m}$ is a $m$-loop contribution. The same no longer holds for $\beta_{n}, n>1$. In general, each coefficient $b_{n, m}$ mixes contributions from different loop orders.
} 
a polynomial of degree $k$ in $(j-2)$. The form of this expansion should be compared with (3.5) for the function $j(\nu, \tau)$. Both expansions make explicit that we are doing a strong coupling expansion around the diffusion limit $j \rightarrow 2$ and $\lambda \rightarrow \infty$ with $\sqrt{\lambda}(j-2)$ fixed.

Let us now relate the expansions (3.14) and (3.16) for the dimension $\Delta_{P}(j, \tau)$, to the expansion (3.5) for the Reggeon spin $j(\nu, \tau)$. For simplicity we consider first the computation of the intercept. Since $\beta_{1}(\lambda, \tau)>0$ the function $j(\nu, \tau)$ has a minimum at $\nu=0(\Delta=2)$. This minimum determines the Pomeron intercept $j_{0}=j(0, \tau)$. Thus, setting $\Delta_{P}\left(j_{0}, \tau\right)=2$ in $(3.14)$ we learn that the intercept is fixed by

$$
0=\left(\Delta_{P}\left(j_{0}, \tau\right)-2\right)^{2}=\tau^{2}+\beta_{1}(\lambda, \tau)\left(j_{0}-2\right)+\beta_{2}(\lambda, \tau)\left(j_{0}-2\right)^{2}+\beta_{3}(\lambda, \tau)\left(j_{0}-2\right)^{3}+\cdots,
$$

with $\beta_{n}$ given by the expansion (3.15). Replacing the expansion for the intercept, as defined by (3.5), It is now possible to find $j_{0}$ iteratively in an expansion

$$
j_{0}=2+\frac{c_{1}}{\lambda^{1 / 2}}+\frac{c_{2}}{\lambda}+\frac{c_{3}}{\lambda^{3 / 2}}+\frac{c_{4}}{\lambda^{2}}+\cdots
$$

Note that the coefficients $c_{i}$ are already defined in the expansion (3.5) of the Reggeon spin function, more precisely we have $c_{1}=-\tau^{2} / 2$ and $c_{n}=c_{1} c_{n, 0}$ for $n \geq 2$. Substituting (3.19) and (3.15) into (3.18), and collecting all terms in powers of $1 / \sqrt{\lambda}$, one can determine $c_{n}$ iteratively. To illustrate how this goes, we list here the first few coefficients (note that we set $\left.b_{10}=1\right)$,

$$
\begin{aligned}
c_{1}= & -\tau^{2} / 2, \\
c_{2}=- & b_{1,1} c_{1}, \\
c_{3}=- & {\left[b_{1,1} c_{2}+b_{1,2} c_{1}+b_{2,0} c_{1}^{2}\right], } \\
c_{4}=- & {\left[b_{1,1} c_{3}+b_{1,2} c_{2}+b_{1,3} c_{1}+2 b_{2,0} c_{1} c_{2}+b_{2,1} c_{1}^{2}\right], } \\
c_{5}=- & {\left[b_{1,1} c_{4}+b_{1,2} c_{3}+b_{1,3} c_{2}+b_{1,4} c_{1}+b_{2,0}\left(c_{2}^{2}+2 c_{1} c_{3}\right)+2 b_{2,1} c_{1} c_{2}+b_{2,2} c_{1}^{2}+b_{3,0} c_{1}^{3}\right], } \\
c_{6}=- & {\left[b_{3,1} c_{1}^{3}+b_{2,3} c_{1}^{2}+3 b_{3,0} c_{2} c_{1}^{2}+b_{1,5} c_{1}+2 b_{2,2} c_{2} c_{1}+2 b_{2,1} c_{3} c_{1}+2 b_{2,0} c_{4} c_{1}+b_{2,1} c_{2}^{2}\right.} \\
& \left.+b_{1,4} c_{2}+b_{1,3} c_{3}+2 b_{2,0} c_{2} c_{3}+b_{1,2} c_{4}+b_{1,1} c_{5}\right] .
\end{aligned}
$$

In the diffusion limit, the intercept reduces to $\alpha_{P}(\tau) \equiv j_{0}(\tau)=2-\tau^{2} / 2 \sqrt{\lambda}$, as stated above. As for the leading twist case, $\tau=2$, this intercept corresponds to the location of a square-root branch point for the spectral curve $\Delta_{P}(j, \tau)$, and it approaches $j=2$ in the limit of $\lambda \rightarrow \infty$.

More generally, we can relate the coefficients of the polynomials $H_{k}(j-2, \tau)$ entering the expansion (3.16) of $\Delta_{P}(j, \tau)$, with the coefficients of the polynomials $\tilde{j}_{n}\left(\nu^{2}, \tau\right)$ entering the expansion (3.5) of $j(\nu, \tau)$. These functions are simply related by the inversion formula $\Delta_{P}(j(\nu, \tau), \tau)=2+i \nu$ given in (3.2). This is a mechanical computation, so we only give here the relation between the first coefficients without further explanations (excluding the coefficients 
$c_{n, 0}$ already given above)

$$
\begin{aligned}
c_{3,1}= & b_{2,0} / 2, \quad c_{4,1}=\left[-3 b_{1,1} b_{2,0}+b_{2,1}\right] / 2, \quad c_{4,2}=0, \\
c_{5,1}= & {\left[6 b_{1,1}^{2} b_{2,0}-3 b_{1,2} b_{2,0}+2 \tau^{2} b_{2,0}^{2}-3 b_{1,1} b_{2,1}+b_{2,2}-\tau^{2} b_{3,0}\right] / 2, } \\
c_{5,2}= & {\left[2 b_{2,0}^{2}-b_{3,0}\right] / 4, \quad c_{5,3}=0, } \\
c_{6,1}= & {\left[-10 b_{1,1}^{3} b_{2,0}+12 b_{1,1} b_{1,2} b_{2,0}-3 b_{1,3} b_{2,0}-10 \tau^{2} b_{1,1} b_{2,0}^{2}+6 b_{1,1}^{2} b_{2,1}-3 b_{1,2} b_{2,1}+4 \tau^{2} b_{2,0} b_{2,1}\right.} \\
& \left.-3 b_{1,1} b_{2,2}+b_{2,3}+4 \tau^{2} b_{1,1} b_{3,0}-\tau^{2} b_{3,1}\right] / 2, \\
c_{6,2}= & {\left[-10 b_{1,1} b_{2,0}^{2}+4 b_{2,0} b_{2,1}+4 b_{1,1} b_{3,0}-b_{3,1}\right] / 4, \quad c_{6,3}=c_{6,4}=0 . }
\end{aligned}
$$

\subsection{Explicit results for $\mathcal{N}=4$ SYM}

It is now clear that we can use the known results for the spectral curve $\Delta_{P}(j, \tau)$ for $j \sim 2$ to extract information about the strong coupling expansion Pomeron spin $j(\nu, \tau)$, and in particular to compute the Pomeron intercept for $\tau \geq 2$. We begin by noting that, since $\beta_{1}(\lambda, \tau)=$ $2 \tau \alpha_{1}(\lambda, \tau)$ is known analytically, it can be easily expanded in powers of $1 / \sqrt{\lambda}$, with the result [15]

$\beta_{1}(\lambda, \tau)=2 \lambda^{\frac{1}{2}}\left(1-\frac{1}{2 \lambda^{1 / 2}}+\frac{4 \tau^{2}-1}{8 \lambda}+\frac{4 \tau^{2}-1}{8 \lambda^{3 / 2}}+\frac{-16 \tau^{4}+104 \tau^{2}-25}{128 \lambda^{2}}+\frac{-16 \tau^{4}+56 \tau^{2}-13}{32 \lambda^{5 / 2}}+\cdots\right)$.

From this expansion it is straightforward to extract the coefficients $b_{1, m}$. This is enough to fix $c_{1}$ and $c_{2}$ in the computation of the intercept (3.20), and in particular agrees with the diffusion limit result $c_{1}=-\tau^{2} / 2 .^{11}$

To find the intercept coefficients $c_{n}$ for $n>2$, knowledge of the coefficients $b_{n, m}$ for higher $\beta_{n}$ are required. As mentioned earlier, (3.13) is an expansion with increasingly divergent coefficients, i.e. $\alpha_{n}=O\left(\lambda^{n / 2}\right)$. Clearly, very special cancellations must take place in moving from (3.13) to (3.14) for convergence. Recently, explicit expressions for $\alpha_{2}(\lambda, \tau), \tau=2$ and 3, have been obtained [19]. With the aid of numerical analysis, together with consistency matching with (3.22), [19] also gives strong coupling expressions for arbitrary $\tau$, up to order $\lambda^{-3 / 2}$,

$$
\begin{aligned}
\alpha_{2}(\lambda, \tau)= & -\frac{\lambda}{2 \tau^{3}}+\frac{\lambda^{1 / 2}}{2 \tau^{3}}+\frac{1}{4 \tau}+\frac{1-\tau^{2}(24 \zeta(3)+1)}{16 \tau^{3} \lambda^{1 / 2}}-\frac{8 \tau^{4}+\tau^{2}(72 \zeta(3)+11)-4}{32 \tau^{3} \lambda} \\
& +\frac{24 \tau^{4}(16 \zeta(3)+20 \zeta(5)-7)-48 \tau^{2}(31 \zeta(3)+20 \zeta(5)+7)+75}{256 \tau^{3} \lambda^{3 / 2}}+O\left(\lambda^{-2}\right) .
\end{aligned}
$$

It is then possible to calculate expansions for $\beta_{2}$, with relevant low order coefficients $b_{2, m}$ extracted from the expansions of $\alpha_{1}$ and $\alpha_{2}$,

$$
\begin{aligned}
\beta_{2}(\lambda, \tau)=2 & \left(\frac{3}{4}-\frac{3 \zeta(3)-3 / 8}{2 \lambda^{1 / 2}}-\frac{\tau^{2}+9 \zeta(3)-5 / 8}{4 \lambda^{3 / 2}}\right. \\
& \left.+\frac{\tau^{2}(3 \zeta(3)+15 \zeta(5) / 4-27 / 16)-15 \zeta(5) / 2-93 \zeta(3) / 8-3 / 16}{2 \lambda^{2}}+\cdots\right) .
\end{aligned}
$$

\footnotetext{
${ }^{11}$ The all coupling expansion carries more information about the coefficients $c_{n, k}$ of the $j(\nu, \tau)$ expansion (3.5). Indeed, it is simple to check that the combination $\sum_{k=0}^{n-2}(-1)^{k} \tau^{2 k} c_{n, k}$ is entirely fixed by the coefficients $b_{1, m}$ with $m<n$.
} 
The expansions of $\beta_{n}$ for $n>2$ are currently not known to high order. However, as discussed in section 6.3 of [19], from an analysis of classical energy, with semi-classical corrections, it is in principle possible to extract the $\tau$-independent coefficients $b_{n, 0}$ and $b_{n, 1}$ for all $n$. For our calculation of the intercepts we use $b_{3,0}=-3 / 16$ and $b_{3,1}=(60 \zeta(3)+60 \zeta(5)-17) / 32$. Note that all coefficients $b_{n, m}$ are polynomials in $\tau$, regular at $\tau=0$. We have also identified, for each coefficient, its order in a string loop-expansion [35-37]. With these coefficients it is possible to fix the $c_{n}$ up to $n=6$. Thus, from (3.20), we find that the generalized Pomeron intercept is given by

$$
\begin{aligned}
\alpha_{P}(\lambda, \tau)=j_{0}(\lambda, \tau)= & 2-\frac{\tau^{2}}{2 \lambda^{1 / 2}}-\frac{\tau^{2}}{4 \lambda}+\frac{\tau^{2}\left(-3+\tau^{2}\right)}{16 \lambda^{3 / 2}}-\frac{\tau^{2}\left[-12+\tau^{2}(11+24 \zeta(3))\right]}{64 \lambda^{2}} \\
& +\frac{\tau^{2}\left[-63+6 \tau^{2}(19+48 \zeta(3))-2 \tau^{4}\right]}{256 \lambda^{5 / 2}} \\
+ & \frac{\tau^{2}\left[-216+\tau^{2}(637+1536 \zeta(3)+480 \zeta(5))-2 \tau^{4}(17+36 \zeta(3)+60 \zeta(5))\right]}{512 \lambda^{3}}+\cdots .
\end{aligned}
$$

For $\tau=2$ notice that the $\zeta(5)$ term is absent from $c_{6}$ due to cancellation, but it is in general present. We will show in the next section that just by varying $\tau$, we can use this equation to calculate the Odderon intercept to the same order as above.

We may also compute, with the above information, the remaining coefficients $c_{n, k}$ up to $n=6$, by using (3.21). Such non-vanishing coefficients are $^{12}$

$$
\begin{aligned}
& c_{3,1}=\frac{3}{8}, \quad c_{4,1}=3 \frac{7-8 \zeta(3)}{32}, \quad c_{5,1}=\frac{59-144 \zeta(3)-2 \tau^{2}}{64}, \quad c_{5,2}=\frac{21}{64}, \\
& c_{6,1}=\frac{291-480 \zeta(5)-76 \tau^{2}-48 \zeta(3)\left(32+7 \tau^{2}\right)}{256}, \quad c_{6,2}=\frac{137-204 \zeta(3)-60 \zeta(5)}{128} .
\end{aligned}
$$

\section{Conformal Odderon}

It is appropriate to begin by first mentioning that the importance of Pomeron lies partly in the fact that all high energy hadron-hadron total cross sections $\sigma_{T}$ continue to rise from collider to cosmic ray energies. This universal behavior can be understood as driven initially by the leading $1 / N_{c}$ power law growth, $\sigma_{T} \sim s^{\alpha_{P}-1}$ for the Pomeron intercept $\alpha_{P}>1$. Eventual agreement with the Froissart bound $\sigma_{T} \sim \log ^{2} s$ requires a re-summation of higher order terms in $1 / N_{c}^{2}$ expansion. ${ }^{13}$ The importance of the leading $C=-1$ component, generically referred to as the Odderon [43-46], lies in the fact that it contributes to the difference of the antiparticle-particle and particle-particle total cross sections, $\Delta \sigma_{T}(s) \sim s^{\alpha_{O}-1}$.

In the weak coupling limit, the Pomeron [7-11] can be associated with 2-gluon exchange whereas Odderons can be thought as a $C=-1$ composite of a three-gloun system [47-53]. Two

\footnotetext{
${ }^{12}$ Actually, since [19] also computes $b_{4,0}=31 / 128$ and $b_{4,1}=(901-5520 \zeta(3)-5120 \zeta(5)-3640 \zeta(7)) / 1024$, we can determine $c_{7,3}=391 / 1024$ and $c_{8,4}=(15081-27120 \zeta(3)-12320 \zeta(5)-3640 \zeta(7)) / 8192$.

${ }^{13}$ One often adopts an eikonal sum. Alternatively, the data is sometimes fitted directly by $\sigma_{T} \sim \log ^{2} s$, the maximally allowed asymptotic term consistent with saturating the Froissart unitarity bound. A more thorough discussion can be found in [20] and references therein.
} 
leading Odderons have been identified. One has an intercept slightly below one [47-49], with $\alpha_{O, a} \approx 1-O(\lambda)$, and the second has an intercept exactly at one, $\alpha_{O, b} \approx 1$, up to third order in the 't Hooft coupling [50]. It has also been suggested recently, for $\mathcal{N}=4 \mathrm{SYM}$, that the latter remains exactly at $j=1$, to all orders in weak coupling $[54,55]$. Interestingly, these correspond nicely with strong coupling analysis in the diffusion limit [20, 56].

Recall that, in a weak coupling BFKL treatment, the spectral curve is obtained by an expansion about $j=1$ in the weak coupling $\alpha_{s}$, i.e., $j \approx 1-\alpha_{s} E_{N}\left(\Delta ;\left\{\ell_{N}\right\}\right)$, where $E_{N}$ can be identified with the spectrum for a system of $N$-reggeon states [57-59], labelled by additional indices $\left\{\ell_{N}\right\}$. For $N=2$, this leads to

$$
j=1+\alpha_{s}\left\{2 \Psi(1)-\Psi\left(\frac{(n+3)-\Delta}{2}\right)-\Psi\left(\frac{\Delta-(n+1)}{2}\right)\right\}
$$

where there is a single index, $n=0,1, \cdots$, labelling the principal series representation of $\operatorname{SL}(2, \mathcal{C})$. The leading Pomeron corresponds to $n=0$. Note that this representation is a perturbation about $j=1$, and the right-hand side develops singularities due to poles of the $\Psi$-function. This representation therefore cannot be extended to the region of large $j$ and $\Delta$. For $N=3$, appropriate for the Odderons, one solution coincides with that for $N=2$, with $n=1$ and $\Delta=2$, leading to an Odderon intercept $j=1$, as indicated above.

In this section, we examine these strong coupling results, going beyond the diffusion limit. In particular, we clarify how for the special Odderon solution, $\alpha_{O, b}=1$ at $k=0$, can hold to all order in $1 / \sqrt{\lambda}$.

For the Odderon, the large $\lambda$ difusion limit corresponds to setting $\lambda \rightarrow \infty$ and $j \rightarrow 1$, with $\sqrt{\lambda}(j-1)$ fixed. In this limit the Odderon propagator can be obtained by perturbing about the EOM for the anti-symmetric Kalb-Ramond field, $B_{M N}$ in AdS: $\left(-\square_{\text {Maxwell }}+m_{\text {ads }}^{2}\right) B_{M N}=0$. Here $\square_{\text {Maxwell }}$ stands for the Maxwell operator. Its exact form can be found in [20], and it can again be diagonalized in terms of $O(4,2)$ Casimir. The result is that all modes with odd positive integral $j$ contribute to the exchange, and one arrives at an effective propagator in the $\nu^{2}-j$ plane of the form

$$
G_{O}(j, \nu) \sim \frac{1}{\nu^{2}+m_{\mathrm{AdS}}^{2}+2 \sqrt{\lambda}(j-1)} .
$$

As for the Pomeron, the relevant string modes can be represented by on-shell world-sheet Reggeon vertex operators $\mathcal{V}_{O}^{ \pm}$in AdS. The on-shell condition, $L_{0} \mathcal{V}_{O}^{ \pm}=\bar{L}_{0} \mathcal{V}_{O}^{ \pm}=\mathcal{V}_{O}^{ \pm}$, in analogy with (3.2), leads to $\Delta_{O}(j)=2+i \nu$. It follows that the Odderon spectral curve in the strong coupling diffusion limit, given by the pole locations of $G_{O}(j, \nu)$, is

$$
\left(\Delta_{O}(j)-2\right)^{2}=m_{\text {AdS }}^{2}+2 \sqrt{\lambda}(j-1) .
$$

Denoting $\alpha_{O}(\lambda)$ for the Odderon intercept, it follows that

$$
\Delta_{O}(j)=2+\sqrt{2} \lambda^{1 / 4} \sqrt{j-\alpha_{O}},
$$


where in the diffusion limit

$$
\alpha_{O}(\lambda)=1-\frac{m_{\text {AdS }}^{2}}{2 \sqrt{\lambda}}
$$

We also stress that because of super-symmetry the anomalous dimension at $j=1$ is zero, more precisely, $\Delta_{O}(1)=2+m_{\text {AdS }}$, for any value of the coupling. In the diffusion limit, the spectral curve $\Delta_{O}(j)$ is again parabolic.

From the SUGRA modes, we see that there are two sets of solutions, a set with $m_{\text {AdS,a }}^{2}=$ $(4+k)^{2}$ which we dub as set (a), and a set (b) for which $m_{\text {AdS,b }}^{2}=k^{2}$ with $k=0,1,2, \cdots$. For $k \neq 0$, these modes can be associated with fluctuations in $S^{5}$. For the $k=0$ mode of set (b), at $j=1$, it is known that it can be gauged away since its coupling is through the field-strength which vanishes [60-62]. However, in the diffusion limit of $\lambda$ large but finite, one moves away from $j=1$ with the perturbation introducing an effective AdS mass so that the field-strength no longer vanishes and the mode is now physical. We will return later to discuss this mode further. For each mode $k$ of the $S^{5}$, the two distinct AdS masses directly lead to two distinct Odderon trajectories with intercept given by (4.5) with the associated mass,

$$
\alpha_{O}(\lambda)=1-\frac{m_{\mathrm{AdS}, \mathrm{a} / \mathrm{b}}^{2}}{2 \sqrt{\lambda}}
$$

From the perspective of OPE, at $j=1$, there are two sets of conformal primaries, each indexed by integer $k$, with protected conformal dimensions, ${ }^{14}$

$$
\begin{aligned}
& \Delta_{O}^{(a)}(1)=2+\tau_{a}=2+m_{\mathrm{AdS}, \mathrm{a}}=6+k \\
& \Delta_{O}^{(b)}(1)=2+\tau_{b}=2+m_{\mathrm{AdS}, \mathrm{b}}=2+k .
\end{aligned}
$$

Candidate dual CFT operators dual to these protected string modes are $\operatorname{Tr}\left(F_{\perp \pm} F^{2} Z^{k}\right)+\cdots$ and $\operatorname{Tr}\left(F_{\perp \pm} Z^{k}\right)$, respectively. As usual, one expects that higher spin operators in the leading Regge trajectory can be obtained by acting with derivatives $D^{ \pm}$, leading to operator dimensions $\Delta_{O}^{(a)}(2 n+1)$ and $\Delta_{O}^{(b)}(2 n+1)$ respectively, at $j=2 n+1, n=1,2 \cdots$. However, these can only be obtained meaningfully beyond the diffusion limit.

\subsection{Odderon intercepts in strong coupling}

To go beyond the diffusion limit, let us return to (3.14). In analogy to that equation and (3.16), we expand $\left(\Delta_{O}(j, \tau)-2\right)^{2}$ about $j=1$,

$$
\left(\Delta_{O}(j, \tau)-2\right)^{2}=\tau_{O}^{2}+\beta_{1}^{(-)}(j-1)+\beta_{2}^{(-)}(j-1)^{2}+\beta_{3}^{(-)}(j-1)^{3}+\cdots
$$

We have also added a superscript to the expansion coefficients, $\beta_{n}^{(-)}$, to remind ourselves that we are dealing with the $C=-1$ sector. Recall that (4.9) properly reflects the symmetry in $\Delta_{O} \leftrightarrow 4-\Delta_{O}$. To match the diffusion limit, we require

$$
\beta_{1}^{(-)}\left(\lambda, \tau_{O}\right)=2 \sqrt{\lambda}+O(1) .
$$

\footnotetext{
${ }^{14}$ Of course, each contributes only to correlation functions with appropriate R-charge.
} 
As is the case with the Pomeron, $j\left(\Delta_{O}\right)$ has a minimum at $\Delta_{O}\left(\alpha_{O}, \tau\right)=2$, which defines the Odderon intercept. This, of course, is also equivalent to the existence a square-root singularity, (4.4). We further assume that, as the case for the Pomeron, in the strong coupling limit, the radius of convergence for (4.9) is $O\left(\lambda^{1 / 2}\right)$, and, $\beta_{n}^{(-)}=O\left(\lambda^{(2-n) / 2}\right)$. Correspondingly, we can develop a systematic expansion for $\beta_{n}^{(-)}(\lambda)$ in $1 / \sqrt{\lambda}$,

$$
\beta_{n}^{(-)}=2 \lambda^{\frac{2-n}{2}}\left(b_{n, 0}^{(-)}+\frac{b_{n, 1}^{(-)}}{\lambda^{1 / 2}}+\frac{b_{n, 2}^{(-)}}{\lambda}+\frac{b_{n, 3}^{(-)}}{\lambda^{3 / 2}}+\cdots\right) .
$$

We are now in the position to carry out a similar analysis for Odderon intercepts beyond the diffusion limit. Consider the expansion for the intercept

$$
\alpha_{0}(\lambda)=1+\frac{c_{1}^{(-)}}{\lambda^{1 / 2}}+\frac{c_{2}^{(-)}}{\lambda}+\frac{c_{3}^{(-)}}{\lambda^{3 / 2}}+\frac{c_{4}^{(-)}}{\lambda^{2}}+\frac{c_{5}^{(-)}}{\lambda^{5 / 2}}+\cdots
$$

The coefficients in this expansion can be found by solving $\Delta_{O}\left(\alpha_{O}, \tau\right)=2$ iteratively. Observe that the situation in nearly identical to that for the Pomeron. It follows that $c_{i}^{(-)}$are given exactly by the corresponding coefficients $c_{i}$ for the Pomeron intercept, eq. (3.20), with the replacements of $\tau$ by $m_{\mathrm{AdS}}, c_{i}$ by $c_{i}^{(-)}$and $b_{n, i}$ by $b_{n, i}^{(-)}$. For simplicity, we shall drop the superscript in what follows.

In the diffusion limit, our two sets of Odderon solutions are structurally similar. However, there is no particular reason why these two sets remain similar in higher orders and we shall treat them separately in what follows. We shall first consider type-(a), characterized by $\tau_{a}=m_{\text {AdS }}=$ $4+k, k=0,1,2, \cdots$, before treating the case for type-(b). It is worth mentioning again that the all-coupling formula (3.10) was derived from ABA equations, without the so-called wrapping corrections. It is surprising that they do not appear in the present context as one would expect them especially in the small spin limit. The physical motivation for their absence is not fully understood. ${ }^{15}$ Furthermore, it is supposed to hold only for the $s l(2)$ sector for the configuration with "minimum mode numbers", which should correspond to the spectral curve with minimum scaling dimension. It is indeed possible to generalize the solution of ABA for "non-minimum string modes". However, no systematic attempt has been made [40, 41]. Further discussion will be provided in section 5 .

I - Type-(a) Odderons: as pointed out earlier, the $j=1$ mode survives in the supergravity limit, and it can be identified with the protected CFT operator of the type $\operatorname{Tr}\left(F_{\perp \pm} F^{2} Z^{k}\right)+\cdots$. This conformal primary, just as the case for the Pomeron, can formally be considered as a descendent of super-conformal primary in the $s l(2)$ sector. However, there can be many spectral curves emanating from this protected configuration at $j=1$, for $\lambda$ finite. We assume that the all-coupling formula (3.10) for the slope function applies to the set $\Delta_{O, a}$, corresponding to the "minimum twist" set, and we shall proceed to calculate the Odderon intercept to higher orders in $1 / \sqrt{\lambda}$ under this assumption. The validity of this assumption will be examined in the next section.

\footnotetext{
${ }^{15}$ See $[40,41]$ for a discussion and comparison to ABJM theory.
} 


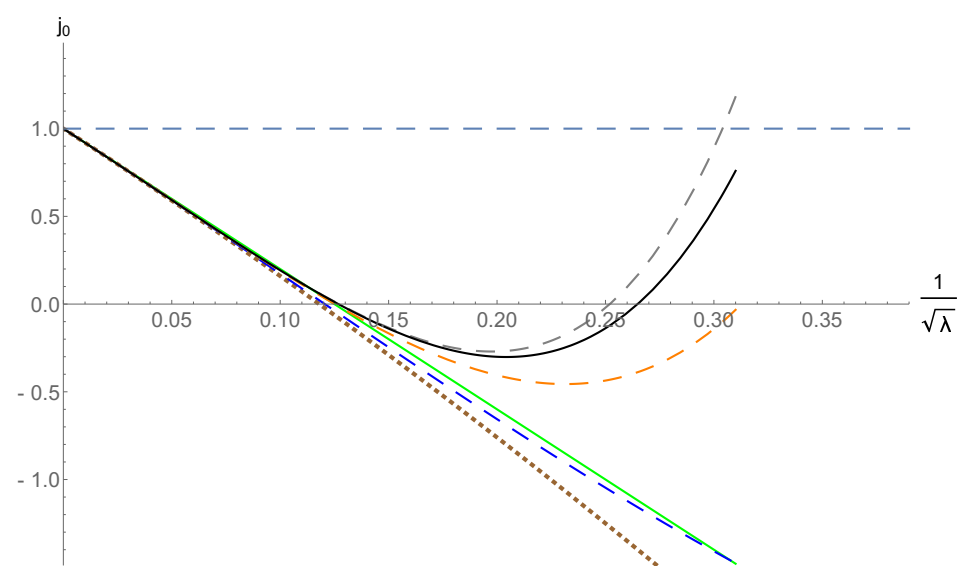

Figure 3. Odderon-a intercept at strong coupling. The solid green-line is to first order in $1 / \sqrt{\lambda}$, the dotted brown-line is to second order, the dashed blue-, orange- and grey-line are to third order, fourth order and fifth order respectively. Finally the solid black-line is the intercept up to sixth order.

That is, we assume that, for type-(a) Odderons, $\beta_{n}\left(\tau_{(a)}\right)=\beta_{n}(4+k)$, with $\beta_{n}$ given by the same functions used for the case of Pomeron. With this we can find the intercept for any $k$, but for simplicity, and to study the case most relevant for QCD, we write the result in the limit $k \rightarrow 0$. Hence $\tau_{(a)}=4$, and we take advantage of the expansion (4.12). Under the assumptions made above, $c_{i}$ can be found by solving the equation

$$
0=4^{2}+\beta_{1}(\lambda, 4)\left(j_{0}-1\right)+\beta_{2}(\lambda, 4)\left(j_{0}-1\right)^{2}+\beta_{3}(\lambda, 4)\left(j_{0}-1\right)^{3}+\beta_{4}(\lambda, 4)\left(j_{0}-1\right)^{4}+\cdots,
$$

iteratively. It is possible to directly adopt the calculation previously done for the Pomeron intercept in section 3 , with $c_{n}$ given by (3.20), by evaluating equation (3.25) at $\tau=4$, after shifting the spin $j$ by 1 . One finds

$\alpha_{O, a}=1-\frac{8}{\lambda^{1 / 2}}-\frac{4}{\lambda}+\frac{13}{\lambda^{3 / 2}}+\frac{96 \zeta(3)+41}{\lambda^{2}}+\frac{288 \zeta(3)+\frac{1249}{16}}{\lambda^{5 / 2}}+\frac{-720 \zeta(5)+192 \zeta(3)+\frac{159}{4}}{\lambda^{3}}+\cdots$.

This intercept $\alpha_{O, a}$ is illustrated in figure 3. Note that coefficients $\left\{c_{n}\right\}$ change signs, with $c_{1}, c_{2}<0, c_{3}, c_{4}, c_{5}>0$, and $c_{6}<0$. We also note that, in the range $0<1 / \sqrt{\lambda}<0.3$, where strong coupling is expected to be useful, the intercept $\alpha_{O, a}$ is below $j=1$. As one increases $1 / \sqrt{\lambda}$ beyond 0.2 , interestingly, it begins to turn around and move towards $j=1$, as it should, only after $c_{4}$ and higher terms are kept. Note as well that the intercept does not continue to blow up, but begins to flatten out as higher orders are taken into account, e.g., with $c_{6}<0$. This behavior fits nicely with the expected matching behavior to first order weak coupling calculation, at $1 / \sqrt{\lambda} \approx 0.3$, as shown in figure 4

Notice that it is a simple exercise to determine the coefficients in an expansion of the type (3.5) for the Odderon spin function $j(\nu, \tau)$.

II - Type-(b) Odderons and $\tau_{b}=k \rightarrow 0$ : as stated earlier, although there are no obvious structural differences between type-(a) and type-(b) Odderon spectral curves in the diffusion 


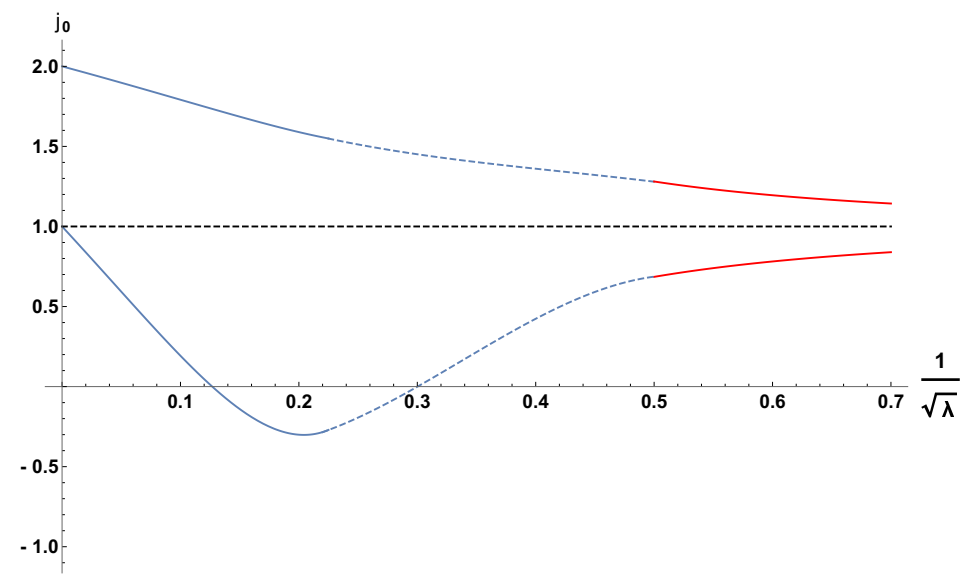

Figure 4. The Pomeron and Odderon-(a) intercepts from strong to weak coupling. The dark blue curves are the calculated strong coupling results, the red curves are the known weak coupling intercepts, and the dashed line is an interpolation. It is interesting to note that up to their current orders, both the Pomeron and Odderon intercepts appear consistent with weak coupling results in the transition region. Black dashed line is for the Odderon-(b) solution where $\alpha_{O, b}=1$.

limit, one crucial difference is the absence of a protected local CFT operator at $j=1$ at $\tau_{b} \rightarrow 0$, in the supergravity limit. For $k \neq 0$, the simplest set of local operators one can identify are $\operatorname{Tr}\left[\left(D^{ \pm}\right)^{j-1} F_{ \pm \perp} Z^{k}\right]$. These operators decouple however in the $k \rightarrow 0$ limit. It is likely that the lowest physical mode on the $k=0$ spectral curve occurs at $j=3$ is $\operatorname{Tr}\left[F_{ \pm \perp} F_{ \pm \perp} F_{ \pm \perp}\right]$ and it is not protected. It has been suggested ${ }^{16}$ that, for $j=3,5, \cdots$, these type-(b) modes could be associated with local operators: $\operatorname{Tr}\left[D_{ \pm}^{j-3} F_{ \pm \perp} F_{ \pm \perp} F_{ \pm \perp}\right]+\cdots$. However, these lead to a different system of $s l(2)$ spin chain, and a separate analysis is required [63-66]. An equally interesting possibility is to consider the sequence $\operatorname{Tr}\left[\left(F_{ \pm \perp} F_{ \pm \perp}\right)^{(j-1) / 2} F_{ \pm \perp}\right]$. Neither sequence leads to well-defined local operators at $j=1$. For this and other considerations, we do not expect the all-coupling formula (3.10) to work for type-(b) Odderon, specially for the $k=0$ mode. Therefore, we shall proceed to carry a more general analysis without assuming the Basso formula for the slope function, $\alpha_{1}$. We shall search for, if any, universal behavior which might survive in the limit of $\tau_{b} \rightarrow 0$.

We proceed by making the expansion for $\beta_{n},(4.11)$, as before, and arrive at

$$
\begin{aligned}
\left(\Delta_{O, b}\left(j, \tau_{b}\right)-2\right)^{2}= & \tau_{b}^{2}+2 \lambda^{1 / 2}\left(1-\frac{b_{1,1}}{\lambda^{1 / 2}}+\frac{b_{1,2}}{\lambda^{3 / 2}}+\frac{b_{1,3}}{\lambda^{2}}+\cdots\right)(j-1) \\
& +2\left(b_{2,0}+\frac{b_{2,1}}{\sqrt{\lambda}}+\frac{b_{2,2}}{\lambda}+\frac{b_{2,3}}{\lambda^{3 / 2}}+\cdots\right)(j-1)^{2} \\
& +2 \lambda^{-1 / 2}\left(b_{3,0}+\frac{b_{3,1}}{\lambda^{1 / 2}}+\frac{b_{3,2}}{\lambda}+\frac{b_{3,3}}{\lambda^{3 / 2}}+\cdots\right)(j-1)^{3} \\
& +O\left((j-1)^{4}\right) .
\end{aligned}
$$

\footnotetext{
${ }^{16}$ B. Basso, private communication.
} 
Note, with the exception of $\tau_{b}=k$ and $b_{1,0}=1$, other coefficients $b_{n, j}$ are unspecified. Expanding $\alpha_{O}$ as in (4.12), after substituting into (4.14), the coefficients $c_{i}$ can be determined iteratively, e.g., leading to formulas essentially given by eq. (3.20). To be more explicit, we can directly express all coefficients $c_{i}$ in terms of $b_{n, i}$. For the first few terms we have, from (3.20),

$$
\begin{aligned}
& c_{1}\left(\tau_{b}\right)=-\frac{\tau_{b}^{2}}{2} \\
& c_{2}\left(\tau_{b}\right)=\frac{\tau_{b}^{2}}{2} b_{1,1} \\
& c_{3}\left(\tau_{b}\right)=\frac{\tau_{b}^{2}}{2}\left(\left(-b_{1,1}^{2}+b_{1,2}\right)-b_{2,0} \frac{\tau_{b}^{2}}{2}\right) \\
& c_{4}\left(\tau_{b}\right)=\frac{\tau_{b}^{2}}{2}\left(\left(b_{1,1}^{3}-2 b_{1,1} b_{1,2}+b_{1,3}\right)+\left(3 b_{1,1} b_{2,1}-b_{2,1}\right) \frac{\tau_{b}^{2}}{2}\right) \\
& c_{5}(\tau)=\frac{\tau_{b}^{2}}{2}\left(\left(-b_{1,1}^{4}+\cdots\right)+\left(-6 b_{1,1}^{2} b_{2,0}+\cdots\right) \frac{\tau_{b}^{2}}{2}+\left(-2 b_{2,0}^{2}+\cdots\right) \frac{\tau_{b}^{4}}{2^{2}}\right) \\
& c_{6}\left(\tau_{b}\right)=\frac{\tau_{b}^{2}}{2}\left(\left(b_{1,1}^{5}+\cdots\right)+\left(10 b_{1,1}^{3} b_{2,0}+\cdots\right) \frac{\tau_{b}^{2}}{2}+\left(10 b_{1,1} b_{2,0}^{2}+\cdots\right) \frac{\tau_{b}^{4}}{2^{2}}\right) .
\end{aligned}
$$

From [19], we expect that $b_{n, j}$ to be polynomials of $\tau_{b}^{2}$. Note that in the limit $\tau_{b} \rightarrow 0$, all coefficients vanish as $\tau_{b}^{2}$, e.g., $c_{1} \sim \tau_{b}^{2}, c_{2} \sim \tau_{b}^{2}$, etc., for arbitrary values for coefficients $b_{n, i}$. It is also easy to verify that, $c_{n+1} / c_{n} \sim O(1)$, for $\tau_{b} \rightarrow 0$. We thus arrive at an important result where, $c_{n}=O\left(\tau_{b}^{2}\right) \rightarrow 0$, for all $n$, in the limit $\tau_{b} \rightarrow 0$. It follows that the leading Odderon intercept for the set-(b), for $\tau_{b}=0$, remains at

$$
\alpha_{O, b}=1
$$

without higher order correction in an $1 / \sqrt{\lambda}$ expansion. This is the long promised result. To state it more graphically, in the limit $\tau_{b} \rightarrow 0$, higher order corrections can change the shape of the spectral curve, without changing its minimum at $\Delta=2$.

For $\tau_{b} \neq 0$, more information is required in order to determine the higher order expansion for their intercepts, e.g., adopting the all-coupling formula (3.10). We will not engage in this exercise here, but note that due to the generality of the above derivation, the $\tau_{b}=0$ result would survive for all possible expansions of $\beta_{n}$.

\section{Conclusions}

We have focussed in this study on the leading $C= \pm 1$ Regge singularities, the Pomeron and Odderon respectively, in strong coupling. Central to our discussion is the notion of spectral curve $\Delta(j)$ for single-trace gauge invariance operators of $\mathcal{N}=4$ SYM. Identifying $\Delta(j)$ in weak coupling remains involved due to possible operator mixings [63]. In strong coupling, spectral curves for leading twist can be identified with bulk degrees of freedom for $D=10$ SUGRA on $A d S_{5} \times S^{5}$ [62]. The Pomeron trajectory can be associated with the Reggeized Graviton, while 
the Odderon trajectories correspond to Reggeized anti-symmetric $A d S_{5}$ Kalb-Ramond tensorfields. With string-vertex operators [5, 20], the relevant single-trace CFT operators and their associated string modes for both Pomeron and Odderon sectors can be identified.

We began by first providing a general discussion on Regge limit in CFT from the perspective of light-cone OPE, and showed how the double-Mellin transforms (2.4) and (2.11) can be used directly in a Minkowski setting. A Regge dictionary (2.13) is established between CFT in coordinate representation and that based on AdS/CFT in a momentum treatment. An important and probably difficult theoretical problem left unresolved is to determine the conditions required in a conformal theory to allow for this representation in the double-Mellin plane. Is this conformal Regge representation a generic property of all $4 \mathrm{D}$ Lorentz invariant conformal theories, or is it restricted to a smaller class of theories?

Due to integrability, these spectral curves can in principle be determined [34], with their inverse $j(\Delta)$ being symmetric under $\Delta \leftrightarrow 4-\Delta$, due to conformal invariance. In this study, we have focussed on "short strings" where each spectral curve takes on a relatively simple form in the large $\lambda$ limit while maintaining the $\Delta \leftrightarrow 4-\Delta$ symmetry. In particular, by adopting the approach advocated in $[14,15,40,41]$, higher order expansion in $1 / \sqrt{\lambda}$ for the Pomerom intercept has been carried out recently $[16,17,19]$. We have generalized this analysis for the Pomeron sector to include non-zero R charge, (3.25), and have also extended the treatment to the case of Odderons, (4.13) and (4.16). For the case of the Pomeron with large classical R charge, it would be interesting to see the appearance of this trajectory in the Regge limit of four-point functions of heavy operators computed in [67] at strong coupling.

It is important to emphasise that our analysis has been carried out in the context of AdS/CFT, appropriate for a strong coupling expansion in the large- $N_{c}$ limit. Simplicity in the complex $\Delta-j$ plane is achieved partly due to the ability to identify modes of SUGRA with protected gauge-invariant YM operators in the limit of $\lambda \rightarrow \infty$, as discussed in section 1 and also in section 4.1. This in turn allows us to treat leading $\Delta(j, \tau)$ curves which dominate the Regge limit through the double-Mellin representation discussed in section 2. It is expected [5] that additional sub-dominate spectral curves exist, leading to "fine-structure" to the complex $\Delta-j$ plane. It is interesting to note in this connection that anomalous dimensions of highertwist Wilson operators in generic gauge theories have previously been investigated and a robust structure, particularly at large $j$, has been found, e.g., a band of trajectories of width growing logarithmically with spin- $j$ [68]. At large- $j$, anomalous dimensions increase with spin as $\ln j$, with leading coefficients given by "cusp-anomalous dimensions". The analysis in [68] was carried out for physical integral $j$-values, in the framework of asymptotic Baxter equation and also based on semiclassical expansion. It is reasonable to expect that this "band-like" structure identified for higher-twist sectors should persist at low- $j$ values, and it is interesting to ask how a smooth connection can be achieved. ${ }^{17}$ Clearly, this can only be discussed meaningfully in the context

\footnotetext{
${ }^{17}$ It is also appropriate to point out that, in the case of a theory with a mass gap, the analytic continuation in $j$ is unique for the partial-wave amplitudes, following what is known as the "Froissart-Gribov" procedure. We
} 
of the large- $N_{c}$ limit where the $j$-plane structure is expected to be simplified, e.g., adopting the approach of quantum spectral curves, advocated in [12, 19]. More immediately, the all coupling analysis in [14, 15, 40,41], which focuses on the small spin region, and is also based on the asymptotic Bethe ansatz, can shed light on this issue.

Since our result depends crucially on the small spin expansion (3.9), it is worth first adding a brief comment on the slope function $\alpha_{1}(\lambda, \tau),(3.10)$, as promised earlier. We first note that the set of gauge invariant operators in the $\operatorname{sl}(2)$ sector, designated symbolically by $\operatorname{Tr}\left(D_{+}^{S} Z^{\tau}\right)$, should be interpreted as a collections of operators, $\operatorname{Tr}\left(D_{+}^{s_{1}} Z D_{+}^{s_{2}} Z \cdots D_{+}^{s_{\tau}} Z\right)$, with $\sum_{j=1}^{\tau} s_{j}=S$. In the large $N$ limit, the dilation operator closes on this subspace, leading to a set of spectral curves, $\Delta_{Z, k}(S, \tau)$, labelled by an index $k$. Our focus here is for strong coupling, where, in the diffusion limit, $\Delta_{Z} \approx \tau+(\sqrt{\lambda} / \tau) S$. Therefore, all these curves are degenerate in this limit. The degeneracy is lifted by going beyond the leading $1 / \sqrt{\lambda}$ limit. Expanding $\Delta_{Z, k}(S, \tau)$ for $S$ small, i.e., $\Delta_{Z, k}(S, \tau)=\tau+\alpha_{1, k}(\tau, \lambda) S+O\left(S^{2}\right)$, these slope functions, $\alpha_{1, k}(\tau, \lambda)$, can be found via ABA, without "wrapping corrections", where the index $k$ can be specified by a set of filling fractions $[15,40,41]{ }^{18}$ The "minimum filling", (or "minimum mode" configuration), leads to the original Basso formula. Clearly, there are many additional lower spectral curves, corresponding to other allowed filling fractions. It would be interesting if these additional spectral curves can be identified with those found at high- $j$ and at weak coupling [63-66, 68]. It is also interesting to find out how these can be related to the mode number in the discussion of GKP strings. In our current treatment, the minimum filling solution has been used for both the Pomeron and the type-(a) Odderon sectors. Note that for both the Pomeron and the type-(a) Odderon keeping the $O(1 / \sqrt{\lambda})$ result for the intercept stemming from the Basso formula agrees with the Pomeron and Odderon intercepts found independently by perturbing about the supergravity limit $[5,20]$.

Let us turn next to the type-(b) Odderon. As we have stated earlier, in the super-gravity limit, the $k=0$ mode decouples at $j=1$, and, for $\lambda$ finite, its physical modes begin at $j=3,5, \cdots$. In section 4.1 , we have carried out a more generally analysis without invoking the all-coupling formula (3.10). Our treatment is based on the structure of large $\lambda$ expansion. Given the diffusion result of $\alpha_{O, b}=1$, for $k=0$, eq. (4.16) follows to all orders. The phenomenon of decoupling also has its counter part in flat space string theory. For $d=4$, Kalb-Ramond tensor field does not lead to a spin-1 massless particle since $B_{\mu \nu}$ has only one independent transverse component. As a 4-d Regge trajectory, its higher string recurrences at $j=3,5, \cdots$ are physical. Therefore, the issue of decoupling can be accomplished by an appropriate vanishing of the coupling, while the dynamics of the whole trajectory remains. (Instead, at $t=0, B_{\mu \nu}$ leads to a spin-0 state, the "axion".) A more detailed analysis will be presented elsewhere.

In this study, we have not fully explored the consequence of super-symmetry, in particular the possibility of more general symmetry patterns for spectral curves $\Delta_{Z}(S, \tau)$. A useful study fully expect a similar procedure can be carried out for generic CFTs, e.g., by adopting, for instance, the Mack representation [32] as a starting point of discussion. As a consequence, there is a unique analytic continuation for $\Delta(j, \tau)$ away from integral $j$ and $\tau$ values. This will be discussed in a future treatment.

${ }^{18}$ To be more explicit, $k \equiv\left\{\kappa_{m}\right\}$, where $\sum_{m \neq 0} \kappa_{m}=1$. Since $\Delta_{Z} \approx \tau+(\sqrt{\lambda} / \tau) S$ in the diffusion limit, it follows that there exists another constraint $\sum_{m}|m| \kappa_{m}=1$. Minimal filling corresponds to $\kappa_{1}=\kappa_{-1}=1 / 2$. 
is a careful examination for the spectral curves for other modes of SUGRA, e.g., scalars, vectors, etc. A preliminary finding involves the possibility of having a more complex structure, e.g., the symmetry about $\Delta=2$ is realized by a pairing of spectral curves, with one symmetric about $\Delta=0$ and the other about $\Delta=4$. Equally interesting is the question for the Odderon intercept in strong coupling from a brane construct alone, without imposing super-symmetry, and its relation to results obtained in weak coupling.

As stressed in [40], $\Delta_{Z}(S, \tau)$ is in general a complicated function of $S, \tau, \lambda$, and also of other quantum numbers. It can in principle possess infinitely many branches, connected through the so-called "crossing-point singularities," i.e., the phenomenon of level-crossings, leading to root-type branch point in $\Delta_{Z}(S, \tau)$. Indeed, in perturbing about the supergravity limit, the multi-valued property of $\Delta_{Z}$ seems to play an important in ensuring $\Delta \rightarrow \Delta-4$ symmetry. It should be stressed that these crossing-point singularities do not lead to branch points for the analytically continued conformal partial-wave amplitude, eq. (2.2). This type of crossing-point singularities has also been noted previously in a weak coupling treatment for Odderons via BFKL-like analysis. As mentioned earlier in section 4, in such a treatment, the spectral curve is obtained by an expansion about $j=1$, i.e., $j \approx 1-\alpha_{s} E_{N}(\Delta ;\{\ell\})$, where $E_{N}$ can be identified with the spectrum for a system of $N$-reggeon states [57-59]. It can be shown that, for $N \geq 3$, level-crossing occurs, leading to crossing-point branch points. However, it is unclear if there is a correspondence for such singularities at weak and strong coupling. Making a precise connection between the strong and weak coupling Odderon solutions remains a challenge. ${ }^{19}$ The approach of quantum spectral curve $[12,19]$ holds the promise of further progress in this direction.

We have focused in this study on the leading planar limit. Note that, in the planar limit, the conformal amplitude growth with a power of $s$, or, equivalently, $1 / \sqrt{u}$, which would violate the flat-space Froissart bound. Clearly, in order to address the issue of Froissart bound for CFT's, one must consider the extension to higher orders in $1 / N^{2}$. One such re-summation is given by the eikonal approximation. From the perspective of light-cone OPE, one must begin including multiple-trace primaries in order to carry out such analysis. It is also interesting to examine the effect of confinement deformation. Since scale invariance is broken in the IR, adopting Poincarepatch for AdS is most suitable for such a treatment. Instead of spectral curves $\Delta(j)$, one now has ordinary Regge singularities $\alpha(t)$ at positive $t$, leading to discrete physical states at integral $j$, e.g., glueballs. One also finds that our double-Mellin representation, (2.4) and (2.11), reduces to a single Mellin (Regge) representation, with a sum over Regge trajectories. These and other related issues will be addressed in future publications.

\section{Acknowledgments}

We would like to thank B. Basso, M. A. Braun, R. de Mello Koch, V. S. Fadin, V. Gonçalves, Y. Hatta, G. Levin, G. Korchemsky, Y. V. Kovchegov, L. Mazzucato, B. Nicolescu, M. Paulos

\footnotetext{
${ }^{19}$ There also exists a slight disagreement [57-59] on the proper interpretation for the Odderon solutions within this weak coupling approach.
} 
and J. Penedones for helpful discussions. R.B., T.R. and C-I.T. would like to thank the Physics Department, University of Porto for hospitality during their visits in the summer of 2014 when this work was completed. The work of R.B. was supported in part by the Department of Energy under Contract No. DEFG02-01ER-40676, that of T.R. and C-I.T. was supported in part by the Department of Energy under Contract No. DE-SC0010010 Task A. The research leading to these results has received funding from the [European Union] Seventh Framework Programme [FP7-People-2010-IRSES] under grant agreements No 269217, 317089, and from the grant CERN/FP/123599/2011. Centro de Fisica do Porto is partially funded by the Foundation for Science and Technology of Portugal (FCT). The work of M.D. is supported by the FCT/Marie Curie Welcome II program.

Open Access. This article is distributed under the terms of the Creative Commons Attribution License (CC-BY 4.0), which permits any use, distribution and reproduction in any medium, provided the original author(s) and source are credited.

\section{References}

[1] J.M. Maldacena, The Large-N limit of superconformal field theories and supergravity, Int. J. Theor. Phys. 38 (1999) 1113 [hep-th/9711200] [InSPIRE].

[2] S.S. Gubser, I.R. Klebanov and A.M. Polyakov, Gauge theory correlators from noncritical string theory, Phys. Lett. B 428 (1998) 105 [hep-th/9802109] [InSPIRE].

[3] E. Witten, Anti-de Sitter space and holography, Adv. Theor. Math. Phys. 2 (1998) 253 [hep-th/9802150] [INSPIRE].

[4] E. Witten, Anti-de Sitter space, thermal phase transition and confinement in gauge theories, Adv. Theor. Math. Phys. 2 (1998) 505 [hep-th/9803131] [INSPIRE].

[5] R.C. Brower, J. Polchinski, M.J. Strassler and C.-I. Tan, The Pomeron and gauge/string duality, JHEP 12 (2007) 005 [hep-th/0603115] [INSPIRE].

[6] A.V. Kotikov, L.N. Lipatov, A.I. Onishchenko and V.N. Velizhanin, Three loop universal anomalous dimension of the Wilson operators in $N=4$ SUSY Yang-Mills model, Phys. Lett. B 595 (2004) 521 [Erratum ibid. B 632 (2006) 754] [hep-th/0404092] [INSPIRE].

[7] L.N. Lipatov, Reggeization of the Vector Meson and the Vacuum Singularity in Nonabelian Gauge Theories, Sov. J. Nucl. Phys. 23 (1976) 338 [inSPIRE].

[8] E.A. Kuraev, L.N. Lipatov and V.S. Fadin, The Pomeranchuk Singularity in Nonabelian Gauge Theories, Sov. Phys. JETP 45 (1977) 199 [INSPIRE].

[9] I.I. Balitsky and L.N. Lipatov, The Pomeranchuk Singularity in Quantum Chromodynamics, Sov. J. Nucl. Phys. 28 (1978) 822 [INSPIRE].

[10] L.N. Lipatov, The Bare Pomeron in Quantum Chromodynamics, Sov. Phys. JETP 63 (1986) 904 [INSPIRE].

[11] R. Kirschner and L.N. Lipatov, Bare Reggeons in Asymptotic Free Theories, Z. Phys. C 45 (1990) 477 [INSPIRE]. 
[12] M. Alfimov, N. Gromov and V. Kazakov, QCD Pomeron from AdS/CFT Quantum Spectral Curve, arXiv:1408.2530 [INSPIRE].

[13] N. Gromov, D. Serban, I. Shenderovich and D. Volin, Quantum folded string and integrability: From finite size effects to Konishi dimension, JHEP 08 (2011) 046 [arXiv:1102.1040] [INSPIRE].

[14] N. Gromov and S. Valatka, Deeper Look into Short Strings, JHEP 03 (2012) 058 [arXiv:1109.6305] [INSPIRE].

[15] B. Basso, An exact slope for AdS/CFT, arXiv:1109.3154 [INSPIRE].

[16] M.S. Costa, V. Goncalves and J. Penedones, Conformal Regge theory, JHEP 12 (2012) 091 [arXiv: 1209.4355] [INSPIRE].

[17] A.V. Kotikov and L.N. Lipatov, Pomeron in the $N=4$ supersymmetric gauge model at strong couplings, Nucl. Phys. B 874 (2013) 889 [arXiv:1301.0882] [INSPIRE].

[18] R.A. Janik and P. Laskoś-Grabowski, Approaching the BFKL Pomeron via integrable classical solutions, JHEP 01 (2014) 074 [arXiv:1311.2302] [INSPIRE].

[19] N. Gromov, F. Levkovich-Maslyuk, G. Sizov and S. Valatka, Quantum spectral curve at work: from small spin to strong coupling in $\mathcal{N}=4$ SYM, JHEP 07 (2014) 156 [arXiv:1402.0871] [INSPIRE].

[20] R.C. Brower, M. Djuric and C.-I. Tan, Odderon in gauge/string duality, JHEP 07 (2009) 063 [arXiv: 0812.0354] [INSPIRE].

[21] R.C. Brower, M. Costa, M. Djuric, T. Raben and C.-I. Tan, Conformal Pomeron and Odderon in Strong Coupling, arXiv:1312.1419 [INSPIRE].

$[22]$ E. D'Hoker and D.Z. Freedman, Supersymmetric gauge theories and the AdS /CFT correspondence, hep-th/0201253 [INSPIRE].

[23] A.V. Kotikov, L.N. Lipatov, A. Rej, M. Staudacher and V.N. Velizhanin, Dressing and wrapping, J. Stat. Mech. 0710 (2007) P10003 [arXiv:0704.3586] [INSPIRE].

[24] L. Cornalba, Eikonal methods in AdS/CFT: Regge theory and multi-reggeon exchange, arXiv:0710.5480 [INSPIRE].

[25] L. Cornalba, M.S. Costa and J. Penedones, Eikonal Methods in AdS/CFT: BFKL Pomeron at Weak Coupling, JHEP 06 (2008) 048 [arXiv:0801.3002] [INSPIRE].

[26] L. Cornalba, M.S. Costa and J. Penedones, Deep Inelastic Scattering in Conformal QCD, JHEP 03 (2010) 133 [arXiv:0911.0043] [INSPIRE].

[27] R.C. Brower, M.J. Strassler and C.-I. Tan, On the eikonal approximation in AdS space, JHEP 03 (2009) 050 [arXiv:0707.2408] [INSPIRE].

[28] R.C. Brower, M.J. Strassler and C.-I. Tan, On The Pomeron at Large 't Hooft Coupling, JHEP 03 (2009) 092 [arXiv: 0710.4378] [INSPIRE].

[29] L. Cornalba, M.S. Costa, J. Penedones and R. Schiappa, Eikonal Approximation in AdS/CFT: Conformal Partial Waves and Finite N Four-Point Functions, Nucl. Phys. B 767 (2007) 327 [hep-th/0611123] [INSPIRE].

[30] L. Cornalba, M.S. Costa and J. Penedones, Eikonal approximation in AdS/CFT: Resumming the gravitational loop expansion, JHEP 09 (2007) 037 [arXiv:0707.0120] [INSPIRE]. 
[31] L. Cornalba, M.S. Costa, J. Penedones and R. Schiappa, Eikonal Approximation in AdS/CFT: From Shock Waves to Four-Point Functions, JHEP 08 (2007) 019 [hep-th/0611122] [INSPIRE].

[32] G. Mack, D-independent representation of Conformal Field Theories in D dimensions via transformation to auxiliary Dual Resonance Models. Scalar amplitudes, arXiv:0907.2407 [INSPIRE].

[33] J.A. Minahan and K. Zarembo, The Bethe ansatz for $N=4$ super Yang-Mills, JHEP 03 (2003) 013 [hep-th/0212208] [INSPIRE].

[34] N. Beisert, C. Ahn, L.F. Alday, Z. Bajnok, J.M. Drummond et al., Review of AdS/CFT Integrability: An Overview, Lett. Math. Phys. 99 (2012) 3 [arXiv:1012.3982] [INSPIRE].

[35] S.S. Gubser, I.R. Klebanov and A.M. Polyakov, A Semiclassical limit of the gauge/string correspondence, Nucl. Phys. B 636 (2002) 99 [hep-th/0204051] [INSPIRE].

[36] S. Frolov and A.A. Tseytlin, Semiclassical quantization of rotating superstring in $A d S_{5} \times S^{5}$, JHEP 06 (2002) 007 [hep-th/0204226] [INSPIRE].

[37] G. Arutyunov, S. Frolov, J. Russo and A.A. Tseytlin, Spinning strings in $A d S_{5} \times S^{5}$ and integrable systems, Nucl. Phys. B 671 (2003) 3 [hep-th/0307191] [INSPIRE].

[38] B.C. Vallilo and L. Mazzucato, The Konishi multiplet at strong coupling, JHEP 12 (2011) 029 [arXiv:1102.1219] [INSPIRE].

[39] L. Mazzucato, Superstrings in AdS, Phys. Rept. 521 (2012) 1 [arXiv:1104.2604] [INSPIRE].

[40] B. Basso, Scaling dimensions at small spin in $N=4$ SYM theory, arXiv:1205.0054 [INSPIRE].

[41] N. Gromov, On the Derivation of the Exact Slope Function, JHEP 02 (2013) 055 [arXiv: 1205.0018] [INSPIRE].

[42] M. Beccaria and G. Macorini, On the one-loop curvature function in the sl(2) sector of $N=4$ SYM, JHEP 06 (2014) 141 [arXiv: 1404.0893] [INSPIRE].

[43] L. Lukaszuk and B. Nicolescu, A Possible interpretation of $p$ p rising total cross-sections, Lett. Nuovo Cim. 8 (1973) 405 [INSPIRE].

[44] G. Bialkowski, K. Kang and B. Nicolescu, High-Energy Data and the Structure of the Odd Signature Amplitude of Pion-Nucleon Scattering, Lett. Nuovo Cim. 13 (1975) 401 [INSPIRE].

[45] J. Finkelstein, H.M. Fried, K. Kang and C.-I. Tan, Forward Scattering at Collider Energies and Eikonal Unitarization of Odderon, Phys. Lett. B 232 (1989) 257 [InSPIRE].

[46] R. Avila, P. Gauron and B. Nicolescu, How can the Odderon be detected at RHIC and LHC, Eur. Phys. J. C 49 (2007) 581 [hep-ph/0607089] [INSPIRE].

[47] J. Kwiecinski and M. Praszalowicz, Three Gluon Integral Equation and Odd c Singlet Regge Singularities in QCD, Phys. Lett. B 94 (1980) 413 [InSPIRE].

[48] J. Wosiek and R.A. Janik, Solution of the odderon problem for arbitrary conformal weights, Phys. Rev. Lett. 79 (1997) 2935 [hep-th/9610208] [INSPIRE].

[49] M.A. Braun, Odderon and QCD, hep-ph/9805394 [INSPIRE].

[50] J. Bartels, L.N. Lipatov and G.P. Vacca, A New odderon solution in perturbative QCD, Phys. Lett. B 477 (2000) 178 [hep-ph/9912423] [INSPIRE]. 
[51] C. Ewerz, The Odderon: Theoretical status and experimental tests, hep-ph/0511196 [INSPIRE].

[52] Y.V. Kovchegov, L. Szymanowski and S. Wallon, Perturbative odderon in the dipole model, Phys. Lett. B 586 (2004) 267 [hep-ph/0309281] [INSPIRE].

[53] A.M. Stasto, Small $x$ resummation and the Odderon, Phys. Lett. B 679 (2009) 288 [arXiv:0904.4124] [INSPIRE].

[54] J. Bartels and G.P. Vacca, Generalized Bootstrap Equations and possible implications for the NLO Odderon, Eur. Phys. J. C 73 (2013) 2602 [arXiv:1307.3985] [INSPIRE].

[55] Y.V. Kovchegov, Running Coupling Evolution for Diffractive Dissociation and the NLO Odderon Intercept, AIP Conf. Proc. 1523 (2012) 335 [arXiv:1212.2113] [INSPIRE].

[56] E. Avsar, Y. Hatta and T. Matsuo, Odderon in baryon-baryon scattering from the AdS/CFT correspondence, JHEP 03 (2010) 037 [arXiv:0912.3806] [INSPIRE].

[57] H.J. de Vega and L.N. Lipatov, Exact resolution of the Baxter equation for reggeized gluon interactions, Phys. Rev. D 66 (2002) 074013 [hep-ph/0204245] [INSPIRE].

[58] S.E. Derkachov, G.P. Korchemsky, J. Kotanski and A.N. Manashov, Noncompact Heisenberg spin magnets from high-energy QCD. 2. Quantization conditions and energy spectrum, Nucl. Phys. B 645 (2002) 237 [hep-th/0204124] [INSPIRE].

[59] G.P. Korchemsky, J. Kotanski and A.N. Manashov, Multi-reggeon compound states and resummed anomalous dimensions in QCD, Phys. Lett. B 583 (2004) 121 [hep-ph/0306250] [INSPIRE].

[60] R.C. Brower, S.D. Mathur and C.-I. Tan, Glueball spectrum for QCD from AdS supergravity duality, Nucl. Phys. B 587 (2000) 249 [hep-th/0003115] [INSPIRE].

[61] R.C. Brower, S.D. Mathur and C.-I. Tan, Discrete spectrum of the graviton in the AdS $S_{5}$ black hole background, Nucl. Phys. B 574 (2000) 219 [hep-th/9908196] [INSPIRE].

[62] H.J. Kim, L.J. Romans and P. van Nieuwenhuizen, The Mass Spectrum of Chiral $N=2 D=10$ Supergravity on $S^{5}$, Phys. Rev. D 32 (1985) 389 [inSPIRE].

[63] A.V. Belitsky, V.M. Braun, A.S. Gorsky and G.P. Korchemsky, Integrability in QCD and beyond, Int. J. Mod. Phys. A 19 (2004) 4715 [hep-th/0407232] [inSPIRE].

[64] A.V. Belitsky, Renormalization of twist - three operators and integrable lattice models, Nucl. Phys. B 574 (2000) 407 [hep-ph/9907420] [INSPIRE].

[65] M. Beccaria, Three loop anomalous dimensions of twist-3 gauge operators in $N=4$ SYM, JHEP 09 (2007) 023 [arXiv:0707.1574] [INSPIRE].

[66] M. Beccaria, G. Macorini and C. Ratti, Wrapping corrections, reciprocity and BFKL beyond the sl(2) subsector in $N=4 S Y M$, JHEP 06 (2011) 071 [arXiv:1105.3577] [InSPIRE].

[67] J. Caetano and J. Toledo, $\chi$-Systems for Correlation Functions, arXiv:1208.4548 [INSPIRE].

[68] A.V. Belitsky, G.P. Korchemsky and R.S. Pasechnik, Fine structure of anomalous dimensions in $N=4$ super Yang-Mills theory, Nucl. Phys. B 809 (2009) 244 [arXiv:0806.3657] [INSPIRE]. 